\title{
La nazione italiana nell'esercito di Alessandro Farnese nei Paesi Bassi: Nuove prostettive
}

\author{
La nazión italiana en el ejércido de Alejandro Farnesio en los Países Bajos: Nuevas \\ perspectivas
}

The Italian Nation in the Alexander Farnese's Army at the Habsburg Netherlands: New perspectives

\section{Giuseppe Bertini}

\author{
Università di Parma ${ }^{1}$
}

Resumen: El ejército multinacional que Alejandro Farnesio dirigió durante su estancia como gobernador en Flandes, tuvo entre sus filas a un elevado número de soldados italianos. Utilizando nueva documentación de archivo, este artículo profundiza sobre su reclutamiento, carreras y empleos.

El trato preferencial que Farnesio otorgó a sus paisanos, le generó problemas con soldados de otras nacionalidades, así como con la corte de Madrid, contribuyendo decisivamente a su cese como gobernador general de Flandes. La biografía de Paolo Rinaldi, escrita para defender la memoria de Farnesio, nos explica el servicio de esos oficiales italianos, así como el comportamiento que Alejandro tuvo con ellos. Gracias a este documento, sabemos que el general trató de conseguir el apoyo de la población local, especialmente de los campesinos, para las operaciones militares, y los italianos, gracias a la estricta disciplina que Farnesio pudo imponerles, le ayudaron a conseguir sus objetivos de un mejor modo que el que pudo conseguir con soldados de otras nacionalidades. Por supuesto, no siempre todas sus expectativas se cumplieron.

Palabras clave: Aprovisionamiento; carrera militar; disciplina; Flandes; Italia; nación; reputación

Abstract: The multinational army, under Alessandro Farnese, had a large presence of Italians. In the light of new archival documentation this paper deals with their recruitment, careers and employment. The preferential treatment accorded by the

\footnotetext{
${ }^{1}$ gbbertini@libero.it
}

(C) 2018 Philostrato. Revista de Historia y Arte 
general to his fellow countrymen created him problems with soldiers of other nationalities and with the Spanish court and contributed greatly to the decision of his removal from power. The biography of Paolo Rinaldi, written to defend the memory of Alessandro, memorials of his officers and their correspondence indicate what was the reason for his behaviour. The general aimed to get the support of the local population, and especially of the peasants, for military operations and Italians, thanks to the stricter discipline he could impose on them, could help him in achieving his objectives, better than soldiers of other nations. Not always his hopes were realized.

Keywords: Procurement; military career; discipline; Habsburg Netherlands; Italy; nation; reputation

Parole chiave: Approvvigionamenti; carriere militari; disciplina; militare; Fiandra; Italia; nazione; reputazione

\section{La nazione italiana nell'esercito asburgico nel Cinquecento}

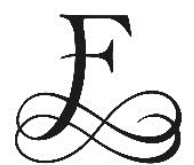

ra il 1578 ed il 1592 l'esercito asburgico, che combatteva nei Paesi Bassi, in Francia e in Germania al comando di Alessandro Farnese, era composto da militari di varie nazionalità: spagnoli, valloni, italiani, tedeschi in primo luogo, ma anche inglesi, scozzesi, irlandesi, borgognoni, lorenesi e albanesi. L'Italia era suddivisa in vari stati; la lingua e la cultura comune, tuttavia, determinavano l'appartenenza di uomini d'arme da essa provenienti alla "nazione italiana", che comprendeva indistintamente sudditi lombardi, napoletani e siciliani di Filippo II, accanto a piemontesi, genovesi, veneti, toscani e a militari originari dallo Stato della Chiesa e dai territori dei Farnese, dei Gonzaga, dei Della Rovere, degli Este ${ }^{2}$. Italiani erano considerati allora i corsi, ma non i sardi, e talvolta uniti ad essi erano gli albanesi, perlopiù cavalleggeri, provenienti da comunità stanziatesi nel Regno di Napoli per sottrarsi al dominio dei turchi che avevano occupato il loro paese. La nazione italiana, la cui compattezza era rafforzata dalla condivisa fedeltà alla chiesa cattolica, si confrontava nei Paesi Bassi con i militari di altri

\footnotetext{
2 Per una definizione di nazione italiana con amplia bibliografia, cfr. Marco Sciarrini, "La Italia natione". Il sentimento nazionale italiano in età moderna, (Milano: F. Angeli, 2004). Ved. anche Alain Tallon (dir.), Le sentiment National dans l'Europe méridionale aux XVI et XVII siècles (France, Espagne, Italie), (Madrid: Casa de Velázquez, 2007), che contiene interessanti contributi di G. Signorotto, A. Prosperi e C. Vasoli. Per un approfondimento del concetto di nazione italiana nel contesto della storia di Spagna, Angelantonio Spagnoletti, "Onore e spirito nazionale nei soldati italiani al servizio della monarchia spagnola", in Militari e società civile nell'Europa dell'età moderna (secoli XVI-XVIII), dirs. Claudio Donati et Bernhard R. Kroener, (Bologna: Il Mulino, 2007), pp. 211-253; Id., "El concepto de naturaleza, nación y patria en Italia y el Reino de Nápoles con respecto a la monarquía de los Austrias", in La Monarquía de las naciones. Patria, nación y naturaleza en la Monarquía de España, dirs. Antonio AlvarezOssorio Alvariño, Bernardo José García García, (Madrid: Fundación Carlos de Amberes, 2004), pp. 505-527. In questo stesso volume, ved. anche Manuel Rivero Rodriguez, "La preeminencia del Consejo de Italia y el sentimento de la nación italiana", pp. 483-503.
} 
paesi, in una rivalità che talvolta assumeva le caratteristiche di aperto conflitto ${ }^{3}$.

Il presente studio si basa, in gran parte, su dati biografici di militari italiani ricostruiti secondo il suggerimento di Léon Van der Essen:

"Un travail des plus utiles consisterait à dresser la liste de tous ces officiers italiens qui, au cours du XVI et du XVII siècle, ont guerroyé dans nos provinces. On y retrouverait, en quantitè, les noms des plus illustres familles de la péninsule, et des états de service éclatants. On y arriverait facilement en dépouillant avec soin les écrivains contemporains des faits qui ont laissé le récit des guerres de Flandre: Bentivoglio, Strada, Campana, Conestaggio, Lanario, Ulloa, Giustiniani...." .

$\mathrm{E}^{\prime}$ necessario tener presente, tuttavia, come famiglie dotate di qualche influenza cercassero di far includere nelle opere storiche dedicate alla guerra i nomi di antenati che avevano ad essa preso parte: Cesare Sardi ricorda il vano tentativo dei lucchesi Guidiccioni per avere le gesta del cavalier Cesare inserite nella storia di Guido Bentivoglio5; ugualmente, a Famiano Strada e ad Angelo Gallucci si richiedeva di far menzione nei loro scritti dei romani Camillo e Torquato Capizucchi ${ }^{6}$. $\mathrm{E}^{\prime}$ da notare, inoltre, come, secondo quanto scrive Carlos Coloma nell'introduzione alla sua opera, gli storici italiani della guerra contro gli stati ribelli al re di Spagna avessero teso ad ampliare il ruolo dei loro connazionali a scapito degli spagnoli ${ }^{7}$. Per ricostruire le biografie e gli stati di servizio dei militari che combatterono nei Paesi Bassi e nei due grandi stati vicini fra il 1578 ed il 1592 si sono, quindi, raccolti dati, oltre che dai lavori storici citati, da corrispondenze conservate in diversi archivi italiani, che permettono di ampliare quanto presentato alla giornata di studio sui "Militari italiani dell'esercito di Alessandro Farnese nelle Fiandre", svoltasi a Fontevivo presso Parma nel $2011^{8}$. Fra le fonti estere che dovrebbero essere oggetto di ulteriori approfondimenti sono i "papeles de servicio", conservati negli archivi della corona spagnola, fatti conoscere da Davide Maffi con la

\footnotetext{
3 Bart De Groof, "Una questione di priorità: spagnoli, italiani e fiamminghi alla morte di Alessandro Farnese", in I Farnese. Corti, guerra e nobiltà in antico regime, dirs. Antonella Bilotto, Piero Del Negro, Cesare Mozzarelli (Roma: Bulzoni, 1997), pp. 167-187.

${ }^{4}$ Leon Van der Essen, Les Italiens en Flandre au XVI et au XVII siècle, (Bruxelles: La Lecture au Foyer, 1926), pp. 26-27.

${ }^{5}$ Cesare Sardi, I capitani lucchesi del sec. XVI, (Lucca: Tipografia Giusti, 1902), p. 94.

${ }^{6}$ Biblioteca Nazionale Centrale Roma (BNCR), Fondo Gesutico, ms. 371, f. 112 e f. 113.

7 Carlos Coloma, Las guerras de los Estados Baxos desde el año de MDLXXXVIII hasta el de MDXCIX, (Amberes: Casa de Pedro y Iuan Bellero, 1625), p. 6: "Los Italianos... quentan nuestras cosas con la tibieza de agenas, dilatandose en las suyas con tanto cuydado, que à quien las leyere sin el, causará alguna duda el determinar la precedencia de ambas naciones, en el valor y disciplina militar".

${ }^{8}$ Questo saggio utilizza ampiamente materiale presentato alla giornata di studio svoltasi a Fontevivo il 24 settembre 2011, i cui atti sono stati pubblicati nel 2013 con importanti contributi di Mario Rizzo, Gianpiero Brunelli, Davide Maffi, Raffaele Tamalio, Alberto Cadoppi e Sebastiaan Derks. Di costante riferimento mi è stato il volume di Gregory Hanlon, The Twilight of a military tradition. Italian Aristocracy and European Conflicts, 1560-1800, (New York: Holmes \& Meier, 1998).
} 
relazione nella citata giornata ${ }^{9}$, e le storie locali delle città dei Paesi Bassi che furono coinvolte in azioni di guerra o in cui furono stanziate guarnigioni di militari italiani ${ }^{10}$.

Il presente lavoro ha utilizzato, inoltre, un'eccezionale fonte rimasta fino ad ora inedita, la biografia di Alessandro Farnese scritta dal suo maggiordomo Paolo Rinaldi, che seguì il principe e poi duca di Parma nel corso dell'intero periodo da lui trascorso nei Paesi Bassi: il testo, datato 1599 e dedicato ai figli Ranuccio ed Odoardo, riflette, con molta probabilità, il pensiero del grande condottiero e ci permette di conoscere i criteri con cui egli conduceva la guerra ed il suo atteggiamento nei confronti dei compatrioti che combattevano nell'esercito asburgico ${ }^{11}$. L'importanza del manoscritto di Rinaldi, ampiamente utilizzato da Famiano Strada e da Léon Van der Essen per la loro ricostruzione del conflitto armato e della personalità del generale, è confermata dal fatto che in esso sono confluite relazioni su singole azioni di guerra che venivano da lui stese contemporaneamente agli eventi" ${ }^{12}$. $E^{\prime} d a$ tener presente come il testo mirasse a difendere la memoria di Alessandro dalle accuse che avevano portato alla sua destituzione ${ }^{13}$ : rappresenta in toni marcatamente negativi chi gli era stato avverso (il duca di Medina Sidonia, Juan Moreo, Pieter-Ernst e Charles Mansfelt, Frédéric Perrenot signore di Champagney) ed è caratterizzato da un forte carattere antispagnolo ${ }^{14}$. Composto negli anni in cui i Farnese, come rilevato da Sebastiaan Derks, avanzavano consistenti rivendicazioni economiche nei confronti della corona

9 Davide Maffi, "Gli uomini del duca. Spunti biografici sull'ufficialità dell'Italia centrosettentrionale al servizio del Farnese", in Militari italiani dell'esercito di Alessandro Farnese nelle Fiandra, dir. Giuseppe Bertini, (Fidenza: Mattioli, 2013), pp. 107-135.

$10 \mathrm{E}^{\prime}$ esemplare la ricerca sul territorio di Hertogenbosch, in cui si ricorda la presenza nel 1580 del capitano romagnolo Camillo Sacchini e la rappresaglia della sua compagnia di valloni contro i contadini, Leo Adriaenssen, Staatsvormend geweld. Overleven aan de frontlinies in de meierij van den Bosch 1572-1629, (Tilburg: Stichting Zuidelijk historisch contact, 2008), pp. 155-156. 11 Per i manoscritti dell'opera, quello della Bibliothèque Royale de Bruxelles, Liber relationum eorum quae gesta fuere in Belgio et alibi per serenissimum D. Ducem Alexandrum Farnesium, ms. II-1135, da cui sono tratte le citazioni qui presentate, e quello della Biblioteca Nazionale Centrale di Firenze, Historia di Fiandra del tempo che comandò I'armata il Duca Alessandro Farnese, ms. II-I-235 (da quest'ultimo si ricavano la data e le dediche), cfr. Sebastiaan Derks, "Le ricompense della guerra: giustificazione e rappresentazione di Alessandro Farnese nel "Liber relationum" di Paolo Rinaldi", in Bertini, Militari italiani, pp. 205-230; Id., "The fruits of war: the representation of Alessandro Farnese in Paolo Rinaldi's Liber Relationum", in Dynastic Identity in Early Modern Eyrope, dirs. Liesbeth Geevers, Mirella Marini, (Farnham: Ashgate, 2015), pp. 153-178.

12 Archivio di Stato di Parma (ASP), Carteggio Farnesiano Estero (CFE), 112 e 113: è del 20 luglio 1583 la relazione della presa di Dunkerque e del 24 maggio dello stesso anno quella della resa di Ypres.

13 Derks, "Le ricompense", pp. 205-230.

14 Rinaldi, Liber Relationum, f. 43v: "Questa nazione spagnola è ben troppo ingorda nel voler dominare, disponere e comandare ogni huomo strapazzando tutto il mondo, la qual cosa è causa di farsi odiosi ogni nazione e in particolare tutti o la maggior parte dei Principi d'Italia, parendo loro come sono Vice Re di Napoli o di Sicilia o Governatori dello Stato di Milano, che ogni uno gli habbia non solo da obbedire, ma adorare, che, come poco pratichi delle cose del mondo, si vengono a far mal volere non solo a Principi con questa loro arroganza, ma a tutto il mondo facendo danno a loro e poco servizio al lor Re". 
asburgica, non fu, probabilmente, pubblicato in quanto poco dopo il suo completamento fu raggiunto l'accordo fra il duca Ranuccio e la Spagna.

\section{Presenza italiana nei Paesi Bassi al tempo di Alessandro Farnese}

Non è semplice determinare la consistenza del contingente italiano nell'esercito di Alessandro Farnese in quanto arrivi e partenze si susseguivano senza sosta. Gli uomini d'arme italiani giungevano inseriti in formazioni militari reclutate nei vari stati della penisola, ma anche singolarmente, accompagnati spesso da lettere di raccomandazione indirizzate al generale da membri della sua famiglia o da chi apparteneva alle loro corti, da sovrani di stati italiani, dal re di Spagna e da suoi ministri ${ }^{15}$. Elevata era la mortalità per malattie ed in battaglia e frequenti erano i rientri determinati da motivi di salute, spesso riconducibili a ferite subite in combattimento, e dai disagi causati dai ritardi nei pagamenti e dalla scarsità di viveri; alcuni uomini d'arme, ricchi dell'esperienza acquisita, ritornavano in Italia per cogliere vantaggiose opportunità d'impiego presso vari stati italiani ${ }^{16}$.

Alessandro, con un piccolo seguito, giunse per la posta a Lussemburgo il 17 dicembre 1577 e un contingente di almeno duecento militari da lui stipendiati arrivò nel gennaio $1578^{17}$. In questo stesso anno Gabrio Serbelloni giunse con un reggimento di duemila fanti reclutati nello stato di Milano ${ }^{18}$. Quando Alessandro assunse il comando dell'esercito nell'ottobre $1578 \mathrm{vi}$ erano, secondo la stima di Ottavio Gonzaga, la fanteria italiana suddivisa in dodici compagnie e la cavalleria italiana composta di seicento uomini, entrambi guidate da capitani di cui purtroppo non indica i nomi ${ }^{19}$. Un buon numero di militari provenienti dalla penisola erano "venturieri", cioè volontari, che avevano talvolta al loro seguito uomini d'arme da loro stipendiati: nell'assedio di Maastricht nella primavera del 1579 fra di essi morirono, o vi furono feriti, due lombardi (Lodovico Visconti, Agostino Schiaffinati), un romano (Flaminio Delfini), due fiorentini (Vincenzo Machiavelli, Francesco Arrighetti) ${ }^{20}$. Alcuni italiani giunsero nei Paesi Bassi nell'autunno del 1579 per

\footnotetext{
15 Giuseppe Bertini, "Carriere di militari italiani nell'esercito di Alessandro Farnese nei Paesi Bassi: merito o privilegio", in Id., Militari italiani, pp. 171-203.

16 Alessandro Farnese con lettera del 22 febbraio 1584 da Tournai comunicava ad Appio Conti che Carlo Sanvitale chiedeva licenza perché voleva "accomodarsi con i Veneziani", in Biblioteca Centrale Nazionale di Roma (BCNR), Fondo Gesuitico, ms. 371, f. 121.

17 Rinaldi, Liber Relationum, f. 34v: "di li a pochi giorni comparve la sua gente di capitani et soldati vechi ch'erano tutti al numero di 200 a sue provisione et spese con grave costo suo". Alcuni di questi uomini d'arme avevano combattuto sotto di lui anche a Lepanto: Nicolò Cesis, Pier Francesco Nicelli, Ambrogio Grappella, Mammolino Rosati, Camillo Capizucchi, Antonio della Somaglia, Matteo Bigliotti.

18 Famiano Strada, Della guerra di Fiandra, Deca prima, (Roma: Facciotti, 1638), p. 487.

19 Leon Van der Essen, El ejército español de Flandes: 1567-1584, (Yuste: Academia de Yuste, 2008), pp. 251-252 e pp. 279-280. Le ricostruite biografie di uomini d'arme permettono di identificarne alcuni che erano giunti nei Paesi Bassi al tempo del duca d'Alba ed altri che avevano seguito Don Giovanni d'Austria.

${ }^{20}$ Famiano Strada, Della guerra di Fiandra, Deca seconda, (Roma: Eredi di Francesco, 1648), p. 86.
} 
combattere, ma non trovarono impiego in quanto nel trattato di Arras, sottoscritto dal re e dagli stati valloni, era prevista la partenza delle truppe straniere ${ }^{21}$ : abbandonarono, infatti, i Paesi Bassi nell'aprile del 1580 spagnoli ed italiani, guidati da Ottavio Gonzaga ${ }^{22}$. Alessandro era riuscito a trattenere parte della cavalleria italiana ed albanese affidandola a Camillo Del Monte, ma aveva suscitato le proteste del Gonzaga che temeva di perdere prestigio in quanto non era stato consultato al proposito ${ }^{23}$. Quattromila italiani tornarono nei Paesi Bassi nell'agosto 1582, formando due tercios di fanteria agli ordini di Mario Cardoino e di Camillo Del Monte ${ }^{24}$. Non mancava, tuttavia, un flusso in direzione opposta per le difficili condizioni in cui si trovava il paese: una terribile carestia (la cosiddetta "fame di Ninove") colpì l'esercito nel $1582-1584^{25}$ e Ippolito Maria Rossi scriveva da Namur nell'agosto 1584: "Di qui passano molti italiani che se ne fuggono, ma io non so che farne se li farò mettere in prigione, perché non vi è che li voglia dar da mangiare" 26 . Nell'autunno 1584 si recò nei Paesi Bassi il marchese del Vasto, che aveva al suo seguito tre capitani e un giovane nobile urbinate, Claudio Corboli, scelti dal cognato duca di Urbino ${ }^{27}$; con lui tornava alla guerra nei Paesi Bassi anche un esperto militare mantovano, Fabio Gonzaga ${ }^{28}$. Nello stesso anno giunse nei Paesi Bassi Pietro Caetani, accompagnato anch'egli da un capitano del ducato d'Urbino ${ }^{29}$. Dopo aver combattuto in Portogallo arrivarono nel 1584 con raccomandazione reale Ippolito Bentivoglio, Cesare d'Avalos e dalla Spagna, sempre raccomandati da Filippo II, Luca Massimo e Gian Girolamo Doria, che era stato paggio alla corte di Madrid ${ }^{30}$. Conosciamo i nomi dei capitani italiani presenti nell'esercito agli inizi del 1586 grazie al dispaccio da Roma di Lorenzo Priuli al doge: erano divisi nei due tercios del romano Camillo Capizucchi, subentrato a Mario Cardoino, deceduto ${ }^{31}$, e del siciliano Gastone Spinola, subentrato a Camillo Del Monte, che era stato nominato governatore di Breda32; altri otto capitani italiani erano a capo di compagnie di valloni,

21 Archivio di Stato di Modena (ASMo), Carteggio Principi Esteri, 1264/3, il colonnello Guido Sangredali di Gubbio giunto a Maastricht nel 1579 raccomandato dal duca di Ferrara non aveva trovato impiego ed era tornato in Italia.

22 Leon Van der Essen, Alexandre Farnèse, Prince de Parme, Gouverneur Général des PaysBas (1545-1592), II, (Bruxelles: Librairie Nationale d'art et d'histoire, 1934), pp. 258-259.

23 Archivo General de Simancas, Estado, leg. 579, lettera di Ottavio Gonzaga a Filippo II del 29 marzo 1580 da Arlon. La cavalleria italiana ed albanese era stata trattenuta da Alessandro come fosse sua guardia personale, cfr. F. Strada, II, cit., p. 172.

24 Van der Essen, Alexandre Farnèse, III, p. 81.

25 Van der Essen, El ejército, p. 323.

26 Archivio di Stato di Napoli (ASN), Archivio Farnesiano (AF), 1722 bis.

27 Biblioteca Oliveriana Pesaro (BOP), Monumenti Rovereschi (MR), II, ff. 42-43, lettera di Alfonso Felice d'Avalos a Francesco Della Rovere del 12 settembre 1584 da Casale. Alessandro aveva in quell'anno aggregato ai tercios italiane compagnie giunte dall'Italia, cfr. Strada, Deca seconda, p. 304.

28 ASP, CFE, 498, lettere di Scipione Gonzaga al cardinale Alessandro Farnese del 7 e 19 settembre 1584 e lettera di Ferrante Gonzaga a Cosimo Masi dell'8 settembre 1584.

29 Ved. sotto.

30 Strada, Deca seconda, pp. 304-305.

31 I capitani del tercio di Camillo Capizucchi erano Silvio Piccolomini, Alfonso Anadario, Marco Maddaleni, Giovan Battista del Prano, Cornelio Gaparini, Giulio Grimaldi, Antonio Gambaloita, Giovanni Antonio Ferrari.

32 I capitani del tercio di Gastone Spinola erano: Matteo da Crema, Paolo Antonio Lanzavecchia, Camillo Latturia, Bernabò Barbò, Francesco Morosini, Ciocca, Lucio Pallavicino, Enrico Rapetti. 
tedeschi, borgognoni ${ }^{33}$; altri ancora erano capitani di compagnie di cavalli (14, di cui 3 albanesi) ${ }^{34}$; vi erano, inoltre, due compagnie di fanteria che erano nei Paesi Bassi da prima dell'arrivo dei tercios, ed una di esse aveva come capo il napoletano Fabio Mataloni ${ }^{35}$. In considerazione dei vuoti creatisi nei due tercios italiani ${ }^{36}$, Alessandro incaricò nel 1587 Biagio Capizucchi di recarsi in Italia per effettuare leve di soldati nello Stato della Chiesa e nel Ducato di Urbino ${ }^{37}$ e nello stesso anno arrivò nei Paesi Bassi un tercio di militari napoletani guidati da Carlo Spinelli, descritti da Vázquez come "muy bizarros y con muchas galas", che furono inviati di guarnigione a Grave, Venlo, Diest e Roermond ${ }^{38}$. Ercole Gonzaga stimava nel 1588, poco prima della prevista spedizione in Inghilterra, che nei tre terzi italiani si trovassero 7000 militari $^{39}$. La presenza di italiani nel novembre 1588 è indicata in una lettera del veneto Fulvio Della Nave: tre tercios di fanteria di 3000 (su un totale di 28300) uomini al comando di tre maestri di campo, Camillo Capizucchi, Gastone Spinola e Carlo Spinelli, i primi due composti da quindici compagnie ciascuno ed I'altro da venti; la cavalleria italiana era costituita da 800 cavalieri suddivisi in nove compagnie sotto i seguenti capitani: Camillo e Francesco Del Monte, Mario Martinengo, Francesco Biumi (Corradino), Annibale Gonzaga, Pietro Caetani, Biagio Capizucchi, Ippolito Bentivoglio, Appio Conti; vi era inoltre la compagnia della guardia di Alessandro Farnese al comando del piacentino Pier Francesco Nicelli e una compagnia di archibugieri guidata dal "castellano di Piacenza" (Leoluca Haller, che forse già allora si trovava in Italia $)^{40}$. Contemporaneamente, per iniziativa del

33 Erano Camillo Sacchini, Matteo Corvini, Ruggero Veronici, Prospero Barozzi, Filippo Rinuccini,
Giovan Francesco Tucci, Camillo Manelli, Troiano Francucci.
34 Erano Camillo Del Monte, Francesco Del Monte, Nicolò Cesis, Appio Conti, Biagio Capizucchi,
Pietro Caetani, Annibale Gonzaga, Mario Martinengo, Ambrogio Landriani, Francesco Biumi,
Pier Francesco Nicelli; gli albanesi erano Nicolò e Giorgio Basti, Giorgio Crescia.
35 Bertini, Militari italiani, Appendice I, pp. 231-237: per quattro o cinque anni le due
compagnie di italiani erano state guidate da Gastone Spinola.
36 La necessità di nuove reclute nelle compagnie italiane è documentata in Francisco Verdugo,
Li commentari delle cose successe in Frisia (Napoli: Nella Stamperia di Felice Stigliola, 1605),
pp. 244-245: "Vi erano eziandio alcune compagnie di cavalli sotto la condotta di D. Alfonso
d'Avalos, fratello del Marchese del Vasto, che tra tutti non arrivavano a cento, essendo le dette
compagnie molto esauste di gente et quasi disfatte et in particolare ve n'era una che non
haveva altro che due archibugieri di vanguardia, tre lance di battaglia et tre femine dishoneste
per retroguardia et quello che era peggio non portavano pur un danaro". Il passo è riportato
in Van der Essen, El ejército, p. 104 . 37 BNCR, Fondo Gesuitico, ms. 131, ff. 103-105v, "Copia dell'instruttione di quello che il Capitano Biagio Capizucchi nel viaggio che deve fare per Italia in servitio di S. M. per conto della leva della infanteria italiana che s'ha da fare per impire li due terzi di essa che qui si ritrovano". Un'altra copia del documento si trova nella Bibliothèque Royale de Bruxelles (BRB), ms. II, 3334, descritto in Leon Van der Essen, "Inventaire analytique d'un recueil de lettres inédites échangées par Alexandre Farnèse avec des membres de la famille Capizucchi (15721592)", in Bulletin de la Commission royale d'Histoire, XCV, (1931), pp. 73-92.

38 Alonso Vázquez, Los sucesos de Flandes y Francia del tiempo de Alejandro Farnese, (Madrid: Colección de documentos inéditos de la historia de España (CODOIN), 1879), 73, pp. 313-314.

39 Archivio di Stato di Mantova, Archivio Gonzaga, 574, lettera di Ercole Gonzaga al duca da Bruges dell'8 luglio 1588, pubblicata in Raffaele Tamalio, "I Gonzaga e le guerre di Fiandre", in Bertini, Militari italiani, pp. 149-150. Il numero dei venturieri era di 4.000 secondo la sua stima, ma non indica quanti di essi fossero italiani.

40 La lettera del 30 novembre 1588, diretta al religioso di Mestre Giulio Cesare Valentino e conservata a Roma nella Biblioteca Vallicelliana, è pubblicata in Gisbert Brom, Archivalia in 
banchiere genovese Tommaso Fieschi, giungevano nei Paesi Bassi 450 corsi al comando di Pompeo Giustiniani. Arrivarono allora come venturieri, in previsione dello sbarco in Inghilterra, anche due membri di famiglie sovrane italiane, Giovanni de' Medici e Amedeo di Savoia, il quale aveva un seguito di 34 uomini $^{41}$. Le scarse opportunità di carriera inducevano molti soldati esperti a lasciare i Paesi Bassi, anche senza l'autorizzazione del generale ${ }^{42}$. Nel 1590 al rientro della spedizione in Francia il duca era accompagnato da sei compagnie di cavalli, comandate, le due della sua guardia da Pier Francesco Nicelli, e le altre da Mario Farnese, Nicolò Cesis, Appio Conti e Biagio Capizucchi, chiamate dai militari delle altre nazioni le "favorite", forse in quanto Alessandro le impiegava nelle azioni che davano maggior prestigio ${ }^{43}$. Nel 1591 alcuni capitani, fra cui Appio Conti, Pietro Caetani, Rodolfo Baglioni, ritornarono in Italia per entrare a far parte dell'esercito papale che Gregorio XIII inviava in Francia in soccorso della Lega al comando del nipote Ercole Sfondrato duca di Montemarciano ${ }^{44}$ e nello stesso anno si recarono nei Paesi Bassi il bolognese Marescotti con duecento fanti e Tarquinio Capizucchi con quattro compagnie, per un totale di mille fanti, raccolte in Romagna ${ }^{45}$. La morte di Alessandro nel 1592 determinò la partenza di buona parte dei militari che si erano formati sotto di lui ${ }^{46}$. In totale Parker stima che fra il 1582 ed il 1593 circa 16 mila fanti marciassero dall'Italia verso i Paesi Bassi ${ }^{47}$.

La presenza italiana nell'esercito asburgico era completata da medici e cerusici $^{48}$, ingegneri militari ${ }^{49}$, segretari e mastri di casa dei venturieri di maggior lignaggio, religiosi ${ }^{50}$, auditori ${ }^{51}$ e servitori. Nelle "Istruzioni" a Pietro Caetani sono specificate le funzioni che doveva svolgere il segretario: scrivere

Italie belangrijk voor de geschiedenis van Nederland ('S-Gravenhage: M. Nijhoff, 1914), III, pp. 87-91.

${ }^{41}$ Elda Ghiglione, Amedeo di Savoia Marchese di San Ramberto (Firenze: Athenaeum, 2005), p. 64.

42 Fernando González de León, The Road to Rocroi. Class, Culture and Command in the Spanish Army of Flanders, 1567-1659 (Leiden-Boston: Brill, 2009), pp. 73-74.

43 Coloma, Las guerras, p. 98.

${ }^{44}$ Gianpiero Brunelli, "Soldati della scuola vecchia di Fiandra". Nobiltà ed esercizio delle armi nello Stato della Chiesa fra Cinque e Seicento", in Bilotto et alíi, I Farnese, pp. 434-435.

45 Cesare Campana, Delle historie del mondo, II (Venezia: I Giunti, 1607), p. 458 e BNCR, Fondo Gesuitico, ms. 371, f. 111 e f. 113.

${ }^{46}$ Brunelli, "Soldati", p. 435.

47 Geoffrey Parker, The army of Flanders and the Spanish Road 167-1659 (Cambridge: Cambridge University Press, 2004), pp. 237-238; i dati sono riportati in Maffi, "Gli uomini", p. 112.

48 Pennone, Tiberio Delfini, il dottor Simonetta, definito "cerusico eccellente" in Rinaldi, Liber Relationum, ff. 238-238v. Nello stesso manoscritto, f. 138v, è annotato che ogni nazione aveva la sua struttura ospedaliera.

49 Numerosi erano i sudditi pesaresi o urbinati dei Della Rovere, specializzati nell'architettura militare (fra questi Francesco Paciotto, Bartolomeo e Scipione Campi). Per gli architetti militari italiani, cfr. Charles Van den Heuvel, "Papiere Bolwercken". De introductie van de italiaanse stede-en vestingbouw in de Nederlanden (1540-1609) en het gebruik van tekeningen (Alphen aan den Rijn: Canaletto, 1991).

50 Uno strano francescano dei minori conventuali, Francesco da Castelnovo, è ricordato in una lettera di Odoardo Lanzavecchia ad Alessandro Farnese del 18 aprile 1588 da Breda, in ASN, AF, 1722 bis: è descritto come "licenzioso, carnale et puoco zeloso dell'honor d'Iddio et suo". ${ }^{51}$ ASN, AF, 1699bis, lettera di Pomponio Castano a Cosimo Masi del 31 maggio 1585 da Breda: I'auditore si proponeva per scrivere la storia della guerra di Fiandra. 
in Italia per far conoscere i particolari della guerra e acquistare per il suo signore presso vari principi meriti che ne promuovessero la carriera ${ }^{52}$. Rinaldi, inoltre, annota nel 1585 la presenza di 300 marinai genovesi, molto esperti dei mari settentrionali, nelle operazioni navali contro Anversa ${ }^{53}$.

\section{Reclutamento dei militari in Italia}

Nel XVI secolo i Farnese avevano possedimenti in diverse regioni della penisola ed intrattenevano strette relazioni con famiglie di tradizioni militari da cui era possibile trarre uomini d'arme per l'esercito di Alessandro. Erano originari dall'Alto Lazio dove il papa Paolo III aveva creato nel 1537 per il figlio Pier Luigi il ducato di Castro; nel 1538 avevano ottenuto il marchesato di Novara e nel 1545 i ducati di Parma e Piacenza; da Margherita d'Austria avevano ereditato vari feudi in Abruzzo; avevano diritti feudali su località della Campania (Torre Annunziata), della Puglia (Altamura), del Basso Lazio (Procida e Roccaguglielma, allora nel Regno di Napoli). Grazie ai benefici ecclesiastici che facevano capo ai cardinali della famiglia, inoltre, avevano frequenti contatti con comunità siciliane, laziali, romagnole e marchigiane; in ambito romano erano imparentati con alcune delle principali casate del Lazio ed il cardinale Alessandro esercitava il suo patronato nei riguardi di un gran numero di famiglie della regione ${ }^{54}$.

Numerosi militari nell'esercito di Alessandro erano membri di famiglie feudali parmigiane e piacentine ${ }^{55}$ e non pochi erano nobili di stati italiani autorizzati a mettersi al servizio del generale dai rispettivi sovrani, grazie agli stretti rapporti che i Farnese intrattenevano con essi. La duchessa di Urbino, Vittoria Farnese, era figlia di Pier Luigi e sorella del duca Ottavio; con i Gonzaga ebbero amichevoli relazioni fra il 1579 ed il 1583, dall'inizio delle trattative per il matrimonio di Margherita, figlia di Alessandro, con il principe ereditario Vincenzo fino al suo annullamento; il duca di Savoia era cugino della moglie del generale in quanto Emanuele Filiberto e Maria erano nati da figli del re Manuel di Portogallo; nel corso del Cinquecento i Farnese avevano

52 Gianpiero Brunelli, "Con insonnij diversi, et visioni stravaganti di guerre": I'esordio di Pietro Caetani nelle Fiandre", in Bertini, Militari italiani, pp. 75-106. Il figlio del duca di Sermoneta aveva presso di sé Fabio Angelico che svolgeva contemporaneamente le funzioni di segretario e di maggiordomo. Già Ottavio Gonzaga aveva in una sua lettera a Silvio Calandra del 21 luglio 1577 manifestato l'esigenza di avere nei Paesi Bassi chi si occupasse delle corrispondenze, cfr. Giuseppe Bertini, Ottavio Gonzaga di Guastalla. La carriera di un cadetto al servizio della monarchia spagnola (1543-1583), (Guastalla: Biblioteca Maldoti, 2007), pp. 67-69. Il marchese del Vasto aveva alle sue dipendenze fino al 1590 il ferrarese Montecatini, suo mastro di casa e segretario, cfr. Alberto Cadoppi, "Un "macello di huomini da bene". Lettere al duca d'Este dalle Fiandre di militari al servizio di Alessandro Farnese", in Bertini, Militari italiani, p.169.

53 Rinaldi, Liber Relationum, ff. 159, 180v.

54 Brunelli, "Soldati", pp. 421-444.

${ }^{55}$ Fra i feudatari parmigiani si possono ricordare Lucio Pallavicino di Ravarano, Carlo e Stilicone Sanvitale di Fontanellato, Ippolito Maria Rossi di San Secondo, Marcantonio e Orazio Simonetta di Sissa, Diofebo Meli Lupi di Soragna, Ludovico Rangoni di Roccabianca; fra i piacentini Alessandro Sforza di Borgonovo, Bernardino Mandelli di Monticelli, Galvano e Carlo Anguissola di Grazzano, Ottavio Landi di Rivalta. 
tentato di creare alleanze matrimoniali con i Medici e gli Este, che tuttavia furono sistematicamente bloccate da Filippo II. Buone erano le relazioni fra il duca di Parma e le repubbliche di Venezia, Genova e Lucca da cui provenivano un certo numero di venturieri e capitani del suo esercito. E' da notare come i singoli stati italiani avessero interesse a fornire il loro sostegno militare alla Spagna, potenza egemone in Italia, nella guerra contro i ribelli nei Paesi Bassi e ciò favoriva il flusso di uomini d'arme dalla penisola al Nord dell'Europa ${ }^{56}$. In base all'esperienza da lui maturata nel corso della guerra, Alessandro preferiva inserire nel suo esercito uomini d'arme dello Stato della Chiesa, in quanto riteneva che fossero più adatti dei lombardi a sopportare le fatiche della guerra in Fiandra ${ }^{57}$.

I principi italiani gli raccomandavano a loro volta giovani sudditi che volevano "veder la guerra" e non trascuravano di seguirne l'inserimento nell'esercito asburgico come si coglie dalla risposta di Alessandro al duca di Ferrara in cui lodava il comportamento dei militari provenienti dal ducato estense e assicurava che avrebbe sempre offerto loro posizioni onorevoli ${ }^{58}$. Il peggioramento dei rapporti dei Farnese e della Spagna con alcuni sovrani poteva comportare rallentamenti nei reclutamenti in Italia: Sisto $\mathrm{V}$ pose nel 1587 ostacoli a leve di sudditi dello Stato della Chiesa (voleva limitarle alle sole città di Ascoli, Fermo, Perugia e Viterbo), ma le difficoltà vennero superate grazie all'intervento del cardinale Alessandro, il quale aveva obiettato che concentrare la provenienza dei militari da alcuni piccoli centri avrebbe fatto aumentare il rischio di ammutinamenti (probabilmente per la solidarietà esistente fra di essi) ${ }^{59}$. I militari arruolati in questa leva, 4825 in totale, furono suddivisi nelle compagnie dei capitani Orazio Fontana, Camillo Manelli, Guerra Andreoni, Flaminio Francolini, Fioravante Fioravanti, Giacomo Gianfigliazzi ${ }^{60}$, Girolamo Graziani, Silla Barignani, Alessandro Rangoni, Celso Celsi61. Reclutamenti furono effettuati anche nel Regno di Napoli nel 1587 dal viceré conte di Miranda:

"quattromila elettissimi fanti divisi in venti compagnie guidate da nobili napoletani, Cola Maria, Bartolomeo, Baldassar, tutti tre Caraccioli, Alessandro e Camillo de' Monti, Federico d'Afflitto, Cicco di Sangro, Alessandro Brancaccio, Ferdinando Spinello marchese di Zirò,

${ }^{56}$ Angelantonio Spagnoletti, "Le dinastie italiane e la guerra nelle Finadre", in Società e storia, 125, (2009), pp. 423-443.

57 BNCR, Fondo gesuitico, ms. 431, Copia dell'instruttione... , cit. Per i rapporti del ducato di Urbino con la Spagna, e per la sua specializzazione nel fornire capitani e militari, cfr. Gianvittorio Signorotto, "Urbino dell'età di Filippo II", in Felipe II (1527-1598), Europa y la Monarquía Católica, ed. José Martínez Millan, (Madrid: Sociedad Estatal para la Conmemoración de los Centenarios de Felipe II y Carlos V, 1998), pp. 833-879.

${ }^{58}$ ASP, CFE, 112, minuta di lettera di Alessandro Farnese al duca di Ferrara del 31 agosto 1587 da Bruxelles.

${ }^{59}$ Brunelli, "Soldati", p. 433; Bertini, "Carriere di militari", pp. 199-200.

60 Archivio di Stato di Firenze, Mediceo del Principato, 2865, f. 417, lettera di Ranuccio Farnese al granduca del 17 giugno 1587 per richiedere l'autorizzazione alla leva in Toscana di truppe da parte del capitano Giacomo Gianfigliazzi.

61 Ibid., f. 106. 
Gioan Antonio Carafa, Alfonso Palagano, Orazio Marchese marchese di Camarota, Gioan Antonio Spina, et altri moltissimi cavalieri, i quali col tempo pervennero a più sublimi comandi, allora capitani, alfieri et anco semplici soldati sotto il maestro di campo Carlo Spinelli"62.

Le relazioni derivanti dal rapporto feudale facilitavano le leve: in una frammentaria nota conservata nel Fondo Gesuitico a Roma si indicava la capacità di raccogliere un numero elevato di buoni soldati da parte dei conti ferraresi Ercole Bevilacqua ("cavaliere ricco et molto principale, potrebbe fare tre o quattro mila fanti") e Ippolito Bentivoglio che ne avrebbe potuto riunire altrettanti, mentre si stimava che Silvio Piccolomini, feudatario toscano, ne avrebbe potuto reclutare due mila ed il reggiano conte Ercole Vicedomini mille $^{63}$. Per gli arruolamenti effettuati da feudatari nel regno di Napoli sono di grande interesse le notizie fornite da Angelantonio Spagnoletti: è probabile che l'espediente da lui descritto nel corso del Seicento di arruolare carcerati a cui veniva concessa in cambio la libertà si praticasse anche al tempo di Alessandro Farnese ${ }^{64}$.

Nelle leve era importante disporre di fondi per corrispondere la "prestanza", parte dello stipendio anticipato ai capitani, che equivaleva al "Gutgeld" per i militari tedeschi: compiti di finanziare il reclutamento venivano assegnati ai governatori di Milano e ai Vicerè napoletani, oltre naturalmente agli ambasciatori spagnoli di Roma e di Genova: Alessandro nelle sue istruzioni indicava in 1500/1600 scudi la somma da corrispondere ai capitani che avrebbero fornito ai soldati la prima paga e coperto le spese di viaggio ${ }^{65}$.

\section{Caratteristiche e motivazioni dei militari italiani}

Le condizioni dei militari italiani nell'esercito multinazionale asburgico dei Paesi Bassi sono spesso prese in esame dagli ambasciatori veneti: secondo Lorenzo Priuli nel $1576 \mathrm{gli}$ italiani erano "i peggio trattati di tutti gli altri; non perché il soldato italiano non sia riconosciuto per ardito e valoroso, ma perché torna conto $\mathrm{a}^{\prime}$ spagnoli che vogliono dominare, abbassare la nazione italiana..." ${ }^{\prime 66}$. Il timore degli spagnoli che i militari italiani potessero utilizzare I'esperienza acquisita per rivoltarsi contro di loro è espressa, oltre che dagli

\footnotetext{
62 Raffaele Maria Filamondo, Il genio bellicoso di Napoli. Memorie istoriche d'alcuni capitani napoletani, (Napoli: Parrino e Mutii, 1694), p. 107.

63 BNCR, Fondo Gesuitico, ms. 431, ff. 102-102v. La nota, anonima e incompleta, potrebbe essere stata stesa da veneziani in quanto per alcuni dei feudatari si valutava la loro disponibilità a lasciare il servizio nei Paesi Bassi.

64 Spagnoletti, "Onore e spirito nazionale".

65 BNCR, Fondo Gesuitico, ms. 131, ff. 103-105v, "Copia dell'instruttione...", cit.

66 "Relazione di Lorenzo Priuli", in Le relazioni degli ambasciatori veneti al Senato, dir. Eugenio Alberi, (Firenze: Società editrice fiorentina, 1861), p. 246, e continuava: "... quelli spagnoli che ne sanno più di altri è perché hanno militato o sotto capitani italiani o in compagnia di soldati italiani".
} 
ambasciatori veneti, anche nel 1592 dal segretario del re Juan Idiáquez ${ }^{67}$. Considerazioni politiche determinavano a Madrid le autorizzazioni alle operazioni di reclutamento per l'esercito nei Paesi Bassi: I'ambasciatore veneziano Matteo Zani scriveva che Filippo II non permetteva negli anni in cui al comando era Alessandro Farnese che gli italiani superassero nel numero gli spagnoli, temendo che il nipote/generale potesse avvalersi dei suoi connazionali per rendersi signore del paese ${ }^{68}$. $E^{\prime}$ da tener presente, per altro, come in Italia si giudicasse negativamente l'invio di italiani al servizio della Spagna nelle guerre estere, in quanto si indeboliva in tal modo la forza militare della penisola69.

Gli italiani si recavano nei Paesi Bassi per acquistare esperienze di guerra, dato che la penisola era da molti anni pacificata sotto la potenza egemone spagnola: molte famiglie feudali si erano nel corso del secolo precedente e nella prima metà del Cinquecento specializzate nella professione militare e avevano a disposizione corpi di uomini pronti a seguirli quando si recavano in guerra. Al tempo stesso i militari italiani potevano essere motivati a combattere dal desiderio di contrastare gli eretici che non riconoscevano I'autorità della chiesa romana: come "foreign fighters" si univano a correligionari che difendevano la fede dagli attacchi di chi si era allontanato dal cattolicesimo ${ }^{70}$. Rinaldi presenta Alessandro come il campione della chiesa cattolica, il quale aveva

"ridotto all'obbedienza di Santa Cristiana Apostolica Romana tanto gran numero di città, terre, castelli, piazze et luoghi forti con tanta quantità di popoli dove era spento ogni sorte di costume cristiano et fattovi ritornare il culto et celebrar le messe et uffici divini d'uso cattolico, che per molti anni adietro era mancato ogni fede et retto vivere per fallace malignità del diavolo et per i comandamenti di lor ambitiosi governatori et ministri"71,

e ancora, per sottolineare gli obiettivi religiosi che il duca si poneva, scriveva:

"Et perché haveva sempre nel cuore l'amore, la fede, et timor di Dio, faceva sempre ne suoi eserciti tener conto della religione et del culto, facendo sempre seguitar dietro di campo et per tutti i quartier della corte et per tutte le compagnie di fanteria et di cavalleria, una quantità di religiosi, la maggior parte gesuiti, che tenessero conto delle anime

\footnotetext{
67 Leon van der Essen, "Une critique de I'administration d'Alexandre Farnèse aux Pays-Bas par Don Juan de Idiáquez, Secrétaire d'Etat de Philippe II (1592)", in Bulletin de la Commission Royale d'Histoire, C, (1936), pp. 167-180.

68 "Relazione di Matteo Zani", in Albéri, Le relazioni, pp. 379-380.

69 Cfr. Spagnoletti, "Le dinastie italiane", per una rassegna di chi aveva espresso queste opinioni.

${ }^{70}$ Frédéric Perrenot, signore di Champagney, accusava nel 1590 gli italiani presenti nei Paesi Bassi di essere senza religione, ved. Aimé Louis Philemon De Robaulx De Soumoy (dir.), Mémoires de Champagney. Discours sur les affaires des Pays-Bas, 1573-1590, avec notice et annotations, (Bruxelles: Société de l'Histoire de Belgique, 1860), pp. 309-310.

${ }^{71}$ Rinaldi, Liber Relationum, f. 241v.
} 
di tutti i soldati et di tutti quelli che seguitavano il campo, facendovi per tutto celebrare messe et divini uffici ogni dì..."72.

Alcuni italiani del suo esercito erano membri di ordini militari che si prefiggevano la difesa della religione: erano cavalieri di Malta (il romano Fabio Farnese, il lucchese Cesare Guidiccioni, i lombardi Andrea Cicogna e Lodovico Melzi, il napoletano Alfonso d'Avalos, il vicentino Marcantonio Angarani, il siciliano Onorato Maiorana), di S. Stefano (il fiorentino Giovan Francesco Tucci, il lunense Camillo Malaspina), di S. Lazzaro (il lombardo Vespasiano Carcano $)^{73}$. Motivazioni religiose potevano coesistere con interessi familiari, in quanto i successi nel campo di battaglia di molti uomini d'arme potevano favorire le carriere di congiunti inseriti nelle gerarchie ecclesiastiche ${ }^{74}$. Altri erano stati banditi nei loro stati per delitti commessi e si erano recati nelle Fiandre per sottrarsi alle pene loro comminate, come il sopra ricordato fiorentino Giovan Francesco Tucci ${ }^{75}$, il friulano Germanico Savorgnan, il genovese Gerolamo Morchio76, i romani Celso Celsi, Vincenzo Maccarani, Giovan Battista Gottifredi77. Fra coloro che erano venuti nei Paesi Bassi perché colpevoli di atti criminali rientra I'alessandrino Girolamo Guazzotti, che era stato bandito dallo Stato di Milano per I'omicidio di uno studente dell'Università di Pavia, di cui, però, si proclamava innocente ${ }^{78}$. Altri cercavano di sottrarsi ai creditori e ristabilire le loro fortune economiche, come i romani Alessandro e Curzio Caffarelli ${ }^{79}$; Livio Podocataro era profugo da Cipro, dove aveva perso tutto il suo patrimonio ${ }^{80}$.

Un buon numero di venturieri italiani erano figli naturali di importanti signori alla ricerca di un miglioramento della loro pozione sociale con successi ottenuti in campo militare. Consistente era la partecipazione alla guerra dei Gonzaga appartenenti a rami illegittimi, come è stato osservato da Raffaele Tamalio ${ }^{81}$; per quanto riguarda le principali famiglie nobili parmigiane, Stilicone Sanvitale era figlio naturale di Eucherio vescovo di Viviers, e veniva raccomandato ad Alessandro dal capo della famiglia, il nipote Carlo

\footnotetext{
72 Rinaldi, Liber Relationum, f. 242.

73 Il pesarese Pompeo Mazza era cavaliere, ma non sappiamo di quale ordine.

74 Bentivoglio, Malaspina, Del Monte, Conti, Gaetani, Anguissola avevano fratelli o figli che ambivano a cariche ecclesiastiche.

75 Rinaldi, Liber Relationum, f. 173: in seguito al valore dimostrato nell'assedio di Anversa il granduca gli aveva revocato il bando per l'omicidio commesso.

76 Archivio di Stato di Genova, Archivio Segreto, 2789, 355, lettera di Alessandro Farnese al doge del 30 dicembre 1588 con la richiesta che intervenisse a suo favore la "remissione del confino" e potesse rientrare in patria.

77 Brunelli, "Soldati", pp. 427-429.

78 ASP, CFE, 112, "Essamine di Gieronimo Guazzotto".

79 Brunelli, "Soldati", p. 430.

80 ASP, CFE, 613, lettera di Scipione Costanzi ad Ottavio Farnese del 10 ottobre 1571 da Venezia: "Egli [Podocataro] oltre la perdita di otto mila scudi d'entrata et di honoratissimi edifici et della splendida casa che continuamente ha tenuto... è necessitato per liberare i figlioli che sono schiavi di Turchi in Cipro raccomandarsi nelle parti d'Italia, di Francia e di Spagna...". Si chiedeva che i padri predicatori ed altri ecclesiastici sollecitassero nelle chiese una raccolta di fondi a suo favore.

81 Tamalio, "I Gonzaga", pp. 137-152.
} 
Sanvitale ${ }^{82}$; Ippolito Maria Rossi di San Secondo, della guarnigione di Namur, era figlio naturale del conte Troilo, e veniva ricordato dal fratello nella sua corrispondenza con il duca83. Federico Cusani era figlio naturale di un nobile milanese, con il quale i Farnese avevano stretti rapporti ${ }^{84}$.

Le spese per partecipare alla guerra erano particolarmente elevate per chi voleva mantenere alto il prestigio della famiglia con un seguito adeguato di uomini e cavalli: esorbitanti erano nei Paesi Bassi i prezzi degli alimenti e del fieno per chi doveva mantenere cavalli. Da una lettera di Pietro Caetani al padre apprendiamo che il mantenimento suo e del seguito comportava una spesa di 680 scudi al mese ${ }^{85}$; i nobili venturieri dovevano, per aumentare la loro popolarità, tenere tavola imbandita per altri militari: Alessandro era solito farlo con i suoi ufficiali ${ }^{86}$ e si raccomandava che lo facesse anche il cognato Alessandro Sforza ${ }^{87}$. Anche un semplice capitano come Silla Barignani faceva presente nelle sue memorie come non fosse stato pagato per i suoi sette anni di servizio e ci avesse rimesso di tasca sua "molte centinaia di scudi"88. Il marchese del Vasto in una lettera al cognato duca d'Urbino manifestava la sua perplessità nell'accettare la carica di generale della cavalleria per gli enormi costi che avrebbe dovuto sostenere: "niuna cosa poteva essere più dannosa per la mia casa di questa...il servire un anno o due in questi paesi se sarà bisogno lo farò, ma mi pare dura cosa impegnarmi con questo carico anni..."89. La stessa preoccupazione era espressa nel 1591 in una lettera di Clelia Farnese a Cosimo Masi, in quanto il marito Marco Pio intendeva in un anno di grave carestia farsi raggiungere nei Paesi Bassi dai servitori e da dieci o dodici cavalli, con una spesa che avrebbe messo in gravi difficoltà la famiglia: chiedeva, riservatamente, che il cugino Alessandro Farnese lo distogliesse dal suo proposito ${ }^{90}$.

Vi erano militari italiani che, per l'educazione ricevuta alla corte di Filippo II, si identificavano con la nazione spagnola: è questo il caso di Ottavio Gonzaga che Don Giovanni definiva spagnolo fino alle viscere ${ }^{91}$ e che, come scrive Rinaldi, si sentiva superiore al conte Mansfelt in quanto godeva del favore degli spagnoli92. Alcuni provenivano da famiglie che avevano precedentemente servito la monarchia in altri campi di battaglia: Galvano Anguissola di Grazzano era giunto nei Paesi Bassi nel 1579 come paggio di

82 ASP, CFE, 112, lettera di Carlo Sanvitale ad Alessandro Farnese del 2 agosto 1583.

83 ASP, CFI, 145, lettera di Troilo Rossi ad Alessandro Farnese del 9 settembre 1586.

${ }^{84}$ ASP, CFE, 111, lettera di Cosimo Masi a Giovan Battista Pico del 4 novembre 1582 da Ninove.

85 Brunelli, "Soldati", p. 430.

${ }^{86}$ ASP, CFE, 110, lettera di Fabio Farnese al duca Ottavio del 31 maggio 1578 da Namur per sollecitare aumento della provvigione inviata ad Alessandro dalla famiglia.

87 ASP, CFE, 113, lettera di Benedetto Giandemaria a Giovan Battista Pico del 31 giugno 1584.

88 BOP, Olivieri 383, 51, Memorie di Silla Barignani.

89 BOP, MR, 375, XI, lettera del marchese d'Avalos a Francesco Maria della Rovere del 22 giugno 1586, ff. 58-59; la lettera continuava: "dove l'huomo serve senza gusto, senza reputazione et con spesa insostenibile".

90 ASP, CFE, 335, lettera di Clelia Farnese a Cosimo Masi del 13 giugno 1591.

91 Lettera di Don Giovanni a Rodrigo de Mendoza del 1 gennaio 1578, pubblicata in Sir William Stirling-Maxwell, Don John of Austria, (London: Longmans-Green, 1883), II, pp. 451-453.

92 Rinaldi, Liber Relationum, f. 66 e f. $69 \mathrm{v}$. 
Alessandro Farnese, ma il padre Alessandro (1532-1606) aveva combattuto in Piemonte contro i francesi nell'esercito di Ferrante Gonzaga e lo zio con il suo stesso nome, Galvano, era morto nella battaglia delle Gerbe ${ }^{93}$. Il re, come si è visto, raccomandava ad Alessandro nel 1584 nobili italiani, che avevano combattuto per lui in Portogallo o avevano fatto parte della sua corte: Ippolito Bentivoglio, Cesare d'Avalos, Alessandro Acquaviva d'Aragona, Gian Girolamo Doria ${ }^{94}$. La famiglia Caetani era sempre stata filo francese e la partenza di Pietro per combattere in Fiandra nel 1584 rappresentava un importante segnale di cambiamento nel suo schieramento politico ${ }^{95}$.

\section{Carriere di italiani nell'esercito di Alessandro.}

Ottenendo nel 1582 che le formazioni di fanti italiane, anziché guidate da colonnelli, fossero equiparate ai tercios spagnoli, Alessandro aveva sottratto agli ufficiali la nomina dei capitani, ma se l'era riservata per sé ${ }^{96}$. Le carriere degli uomini d'arme di nazionalità italiana nell'esercito potevano essere favorite dai suoi rapporti di parentela: avevano legami di famiglia con lui Fabio e Mario Farnese di Latera, Francesco Sforza di Santa Fiora ${ }^{97}$, Appio Conti ${ }^{98}$, Pietro Caetani ${ }^{99}$, Carlo Sanvitale ${ }^{100}$, Alessandro Sforza ${ }^{101}$, Marco Pio di Savoia ${ }^{102}$ ed il marchese del Vasto che aveva sposato nel 1582 la cugina Isabella Della Rovere ${ }^{103}$. Altri militari erano a lui raccomandati da sovrani di stati italiani: particolarmente numerose sono le lettere di presentazione di giovani sudditi che, come si è detto, desideravano "vedere la guerra" da parte del duca di Ferrara e di quello d'Urbino e della madre; avanzamenti per

93 Università Cattolica di Milano (UCM), Archivio Anguissola di Grazzano, C 100, fasc. 2, in Archivio Visconti di Modrone.

${ }^{94}$ Strada, Deca seconda, p. 304; per Acquaviva, cfr. lettera di Juan Idiáquez ad Alessandro Farnese dal Pardo del 13 aprile 1586, in ASN, AF, 1699 bis.

${ }_{95}$ Maria Antonietta Visceglia, "Non si ha da equiparare I'utile quando vi fosse I'honore". Scelte economiche e reputazione: intorno alle vendite dello stato feudale dei Caetani (1627)", in dir. Id, La nobiltà romana in età moderna. Profili istituzionale e pratiche sociali, (Roma: Carocci, 2001), pp. 203-223; Brunelli, "Con insonnij diversi".

96 González de León, The Road to Rocroi, p. 71; in Alicia Esteban Estringana, "Cabos de guerra: satisfacción de la oficialidad y eficacia bélica en el ejército de Flandes, entre los siglos XVI y XVII", in Los oficiales reales: los servidores del rey en la Monarquia Hispanica, (siglos XVI$X V I I)$, Juan Francisco Pardo Molero y Manuel Lomas Cortes (coords.), (Valencia: Universitat, 2012), pp. 270-271, si cita una lettera di Alessandro al re da Tournai del 16 dicembre 1581 in cui lamenta che le unità italiane fossero guidate da giovani senza esperienza, ma provvisti di soldi per le leve.

97 Francesco Sforza di Santafiora era cugino in quanto la nonna Costanza Farnese era sorella di Pier Luigi.

${ }_{98}$ Appio Conti era figlio di Violante Farnese del ramo di Latera.

99 Paolo III Farnese era figlio di Giovannella Caetani.

100 Carlo Sanvitale era figlio di Gerolama Farnese, che nel 1538 aveva sposato Alfonso Sanvitale.

${ }^{101}$ Alessandro Sforza di Borgonovo aveva sposato Isabella, figlia naturale di Ottavio Farnese e quindi era cognato di Alessandro.

102 Il feudatario di Sassuolo aveva sposato nel 1587 Clelia Farnese figlia del cardinale Alessandro e quindi cugina del generale.

${ }^{103}$ Su Alfonso Felice d'Avalos, IV marchese del Vasto (1564-1593), ved. Maurizio Arfaioli, "Giovan Battista Marino e gli "Alfonsi d'Avalos", in Studi secenteschi, LII, (2011), pp. 405-411. 
congiunti erano sollecitati dal duca di Mantova ${ }^{104}$ e da Onorato Caetani, il quale riteneva che il figlio dovesse entrare nel consiglio di guerra ${ }^{105}$; il rappresentante della Spagna a Torino, il barone Paolo Sfondrati, aveva raccomandato il figlio Ercole, che però non aveva voluto trarre vantaggio delle condizioni favorevoli offertegli da Alessandro ed era rientrato presto in Italia ${ }^{106}$. Un buon numero di militari erano parenti di membri delle corti farnesiane di Parma, di Roma e dell'Aquila ${ }^{107}$. Relazioni non ufficiali intrattenute da Alessandro potevano favorire le carriere di alcuni uomini d'arme o essere ritenute alla base di alcuni avanzamenti: il siciliano Gastone Spinola aveva sposato la sorella di Françoise de Renty, la giovane nobile amante del generale, e Frédéric Perrenot nella sua denigratoria campagna contro Alessandro Farnese riconduceva la promozione a capitano del vicentino Vincenzo Capra, paggio e poi suo aiuto di camera, ad una affezione particolare per lui che aveva portato il generale a preferirlo alla sua favorita ${ }^{108}$. Gli aveva concesso "une des vieilles compagnies données ordinairement à des gens de qualité et pour de grandes services", ma secondo Rinaldi il generale aveva assegnato a Vincenzo Capra il comando della compagnia di Francesco Ventimiglia dopo la perdita di Breda per il suo comportamento coraggioso nel contrastare la resa dell'importante città ${ }^{109}$.

I giovani nobili inviati dalle famiglie a combattere nei Paesi Bassi avevano talvolta necessità di avere accanto a sé esperti uomini d'arme: il marchese del Vasto richiese tre capitani al cognato duca di Urbino ${ }^{110}$, Pietro Caetani aveva presso di sé un altro capitano urbinate Flaminio Bonaventura che il padre aveva voluto lo accompagnasse ${ }^{111}$ ed il duca di Ferrara prospettava ad Alessandro Farnese per il giovane inesperto Annibale Bentivoglio I'affiancamento di un "luogotenente pratico"112. Anche ad Amedeo di Savoia il fratello duca Carlo Emanuele aveva posto accanto l'esperto capitano Carlo Gazino113; Francesco Sforza di Santafiora, a cui Filippo II aveva concesso il titolo di capitano generale delle truppe italiane, aveva "per compagnia e

\footnotetext{
104 Nel 1583 il duca di Mantova aveva raccomandato ad Alessandro la concessione a Ferrante Gonzaga di un reggimento di tedeschi, cfr. Bertini, "Carriere di militari", p. 194.

105 ASP, CFE, 502, lettera di Onorato Caetani ad Alessandro Farnese del 27 febbraio 1586.

106 ASP, CFE, 396, scambio di lettere fra Alessandro Farnese e barone Sfondrati del 1582, cfr. Bertini, "Carriere di militari", pp. 196-197.

107 Il lunense Alessandro Malaspina era figlio di Comparino, maggiordomo di Maria di Portogallo; il lucchese Cesare Guidiccioni era fratello di Alessandro, gentiluomo del cardinal Alessandro Farnese; i trentini Alvise Betta ed un cugino di cui non si conosce il nome erano nipoti di Francesco, presidente del consiglio ducale; I'umbro Alessandro Astasi era nipote del segretario ducale Giovan Battista Pico; i fratelli piacentini Mentuati erano figli di Girolamo, governatore di Parma; il napoletano Giordano Della Marra era stato un gentiluomo di Margherita d'Austria; , il romano Celso Celsi era nipote di Ascanio Celsi, al sevizio del cardinale Alessandro Farnese.

108 Mémoires de Champagney, p. 310: "...pour lequel il semble retirer ses bonnes grâces à Franceline".

109 Rinaldi, Liber Relationum, f. 220v.

110 BOP, MR, 375.

111 ASP, CFE, 592, lettere di raccomandazione del duca e della madre Vittoria Farnese ad Alessandro Farnese del 20 marzo 1584.

112 ASP, CFE, 232, lettera del 28 agosto 1591.

113 Ghiglione, Amedeo di Saboya, pp. 64-69.
} 
guida" un uomo d'arme di valore, Cesare Palazzolo ${ }^{114}$. Un'interessante lettera di Giacomo Magno al cardinale d'Este spiega come avvenisse I'istruzione di Ercole Bevilacqua per volere di Alessandro Farnese da parte del marchese di Renty nell'impresa di Mechelen: lo faceva entrare nei consigli di guerra e gli mostrava "le occasioni che lo inducono a far battere più in uno luogo che in uno altro et altre cose appartenenti al mestiero di guerra secondo li siti", facendosi da lui accompagnare "a riconoscer i forti e luoghi che vol battere veramente"115. Alcuni capitani venivano destinati da Alessandro a istruttori di militari di riguardo: Camillo Capizucchi aveva avuto fra i suoi allievi Amedeo di Savoia, e lo stesso principe Ranuccio ${ }^{116}$.

Ai venturieri che giungevano dall'Italia Alessandro era solito offrire un trattamento (entretenimiento) di 30 scudi al mese: lo concesse ai lombardi Filippo Crivelli117 e Ercole Sfondrati ${ }^{118}$, al veneto Fabio Della Nave ${ }^{119}$, al cipriota Livio Podocataro ${ }^{120}$. Scarse erano per essi le disponibilità di cariche quali quella di capitano di compagnie di connazionali, ma ad alcuni veniva offerta la guida di compagnie di altre nazioni, di valloni (Camillo Sacchini), di tedeschi (Ferrante Gonzaga), di spagnoli (Ambrogio Landriani), di lussemburghesi (Ercole Rizzi). Alessandro suggeriva che per il cognato Sforza sarebbe stato più facile far carriera in una compagnia di fanteria che in una di cavalleria ${ }^{121}$ e a Marco Pio consigliava di aspettare a raggiungerlo nei Paesi Bassi perché non vi erano in quella fase della guerra opportunità di mettersi in mostra, nè cariche vacanti adeguate al suo rango ${ }^{122}$. La scarsità di cariche è ben descritta dal gesuita Adami nel suo elogio dei Capizucchi:

"Onde non havrà a maravigliarsi chiunque nelle Storie di Fiandra altro di cariche fisse e permanenti non ritroverà conferito dal Farnese a Biagio che una compagnia di cento lancie, benchè l'onorasse in diverse occorrenze di altre cariche e comandi molto maggiori. E pure... sì nobile in quei tempi era il titolo di Capitano di Lance in Fiandra che di quello

\footnotetext{
114 Gianpiero Brunelli, Soldati del papa. Politica militare e nobiltà nello Stato della Chiesa (1560-1644), (Roma: Carocci, 2003), p. 118.

115 ASMo, lettera di Giacomo Magno al cardinale D’Este del 31 agosto 1585, in Cadoppi, "Un "macello di huomini da bene", p. 194.

116 Annibale Adami, Elogi storici de' due Marchesi Capizucchi fratelli Camillo e Biagio celebri guerrieri del secolo passato, (Roma: Stamperia della Reverenda Camera Apostolica, 1685), p. 32; Pietro Fea, Alessandro Farnese duca di Parma. Narrazione storica e militare, (Roma: Fratelli Bocca, 1886), p. 484.

117 ASN, AF, 1722bis, lettera del duca di Brunswick a Cosimo Masi del 12 maggio 1588 da Bruxelles: al capitano Filippo Crivelli era stata da Alessandro concesso un trattamento di trenta scudi al mese che non era stato ancora reso effettivo.

118 ASP, CFE, 396, minuta di lettera di Alessandro Farnese al barone Sfondrati del 7 marzo 1582 da Tournai.

119 Brom, Archivalia in Italie, p. 88.

120 Cadoppi, "Un "macello di huomini da bene", p. 162.

121 ASP, CFE, 113, lettera di Benedetto Giandemaria a Giovan Battista Pico del 31 giugno 1584, in cui riferiva il pensiero del generale: "Quando si arriva ad una compagnia di cavalli pare che sia difficile passare più avanti et nella fanteria si può arrivare più tosto a essere maestro di campo".

122 Biblioteca Ambrosiana Milano (BAM), Fondo Falcò Pio di Savoia, V. N. 468, lettera di Alessandro Farnese a Marco Pio dell'8 marzo 1590.
} 
solo andarono fregiati Prencipi e Cavalieri del più chiaro sangue d'Italia, di Fiandra e di Spagna. Anzi molti personaggi, e soldati valorosi, e che altrove ottennero cariche militari cospicue, militarono sotto il Farnese in Fiandra per più anni, senza poter giungere ad esser Capitani d'una Compagnia d'Archibugieri a cavallo e di Lancie o di Fanteria convenendo contentarsi del solo titolo di Venturiere"123.

In ogni caso, a causa degli elevati costi sostenuti per la partecipazione alla guerra, chi aveva combattuto cercava di compensare il danno subito dal patrimonio familiare con la richiesta di pensioni o benefici al re di Spagna, spesso recandosi personalmente a Madrid a sollecitarli. Anche Marco Pio richiedeva alla corte spagnola ricompense per aver combattuto in Fiandra e in Francia "con gran spesa"124 e da una lettera a lui inviata da Madrid si apprende che nel 1594 vi si trovavano contemporaneamente alcuni dei militari che avevano combattuto con Alessandro: erano in attesa di riconoscimenti economici e favori, anche se molto lenta era la risposta della corte spagnola, Francesco Morosini, il conte Carlo Cicogna, Alessandro Malaspina, Gastone Spinola, I'albanese Giorgio Crescia, il napoletano Annibale Macedonia ${ }^{125}$. Le citate carte dell'archivio degli Anguissola di Grazzano mostrano come si costituisse la pratica di documentazione da inviare alla corte spagnola a sostegno di simili richieste: si ricercavano dichiarazioni di superiori o di persone che nell'esercito ricoprivano cariche elevate per attestare la partecipazione ad operazioni militari di rilievo e spesso anche le ferite riportate ${ }^{126}$.

\section{I militari delle varie nazioni nel giudizio di Alessandro Farnese}

La presenza negli eserciti di corpi militari di differenti nazioni comportava ben noti vantaggi: vi era fra loro emulazione che ne aumentava la combattività e chi guerreggiava lontano dal suo paese era meno portato a disertare per raggiungere i familiari, meno restio ad eseguire ordini che penalizzassero connazionali, meno sensibile a offerte di amici e conoscenti per passare al campo avverso. Scrive Rinaldi riflettendo molto probabilmente l'opinione del suo padrone:

"Nessuna nazione in casa sua propria si trovava che fossero soldati perfetti, perché hanno sempre nell'animo fisso i padri, le madri, le mogli, i figlioli, le case et facoltà loro, pungendoli lo stimolo cotidianamente di ritornare ogni ora a rivederli et non pensar ad altro, che essendo lontano si levano lor dalla memoria tutti questi affetti...

\footnotetext{
123 Adami, Elogi storici, p. 103-104.

124 BAM, Fondo Falcò Pio di Savoia, V. N. 467, memoriale di Marco Pio.

125 Ibíd., lettera di Giacomo Ferrari a Marco Pio da Madrid del 26 marzo 1594.

126 UCM, in Archivio Anguissola di Grazzano sono conservati gli attestati del 1599 sullo stato di servizio di Galvano Anguissola stesi da Ambrogio Landriani, "locotenente general de la Cavalleria ligera delli Stati di Fiandra et capitan de lanze spagnole", e dal contador Pedro Coloma.
} 
Insomma io concludo che gli uomini in casa sua sono pigri et lenti, ma lontani sempre s'industriano al valore e alla virtù"127.

La composizione multinazionale dell'esercito rendeva, per contro, più complessa la conduzione delle operazioni di guerra: il generale doveva tener conto nel pianificare l'impiego di contingenti delle varie nazioni (spagnola, vallona, italiana, tedesca), delle loro attitudini a svolgere compiti diversi (assalti di mura, guardie agli accampamenti, presidi alle città, imboscate, difesa di postazioni sul campo di battaglia) ${ }^{128}$. Rinaldi specifica le collocazioni assegnate da Alessandro Farnese alle varie nazioni negli assedi di città e i compiti loro affidati nelle battaglie. Riportando molto probabilmente il pensiero del generale, afferma che i tedeschi erano adatti a fare la guardia e

"di natura gravi sono atti a star forti in un squadrone in campagna aperta, senza disordinarsi et combattere et lasciarsi ammazzare prima che muoversi, che di andare all'assalto di una breccia d'una terra o bastione, o fosso"; i valloni, per contro, erano più abili "a difendersi dentro a una città o terra o dietro a una muraglia, rivellino o trincea..., che andare a un assalto"129

ed erano, inoltre, molto trascurati nelle guardie ${ }^{130}$. Per assaltare le mura delle città assediate italiani e spagnoli venivano dal generale preferiti: nell'attacco a Borgerhout agli spagnoli assegnò la posizione centrale, mentre valloni e tedeschi furono posti ai loro lati ${ }^{131}$. Considerazioni finanziarie, in particolare, determinavano l'impiego dei tedeschi: le truppe di questa nazione facilmente si ammutinavano, devastando il territorio in cui erano stazionate se i pagamenti non erano regolari. Scrive Rinaldi:

"Per comodità et servizio dell' impresa et utilità del Re era[no] meglio gli italiani che i tedeschi, non essendo questa nazione atta alla guerra se non a stare in campagna forti, in un battaglione, son poi poco buoni agl'assalti o a difender terre, ma sono ottimi ammutinatori costando più un reggimento dei loro che due de italiani o d'altre nationi"132.

La rivalità fra le nazioni era in parte suscitata dal diverso trattamento economico e dalle diverse opportunità di carriera a loro riservate: gli spagnoli

127 Rinaldi, Liber Relationum, cit., f. 109.

128 Fea, Alessandro Farnese, p. 475: "Ed in vero, conoscendo per lunga esperienza le virtù e i vizi di tutte quelle milizie, Alessandro adoperava opportunamente ciascuna di esse nelle fazioni alle quali aveva attitudine maggiore. Dei tedeschi soleva dire che erano buoni soldati, atti a guardar perfettamente le piazze, meno degli altri proclivi agli scandali; ma non potersene far nulla se non puntualmente pagati. Dei valloni si serviva volentieri, ma non risulta punto che li stimasse la miglior fanteria del mondo, come si legge in taluni scrittori. Aveva più alto concetto degli spagnoli, che riguardava come il nerbo dell'esercito; ma sia perché li riconosceva orgogliosi e soverchianti, sia perché italiano, preferiva anche a loro gli italiani, a malgrado dell'opinione contraria prima di lui".

129 Rinaldi, Liber Relationum, f. 94v.

130 Ibid., f. 96: nel corso dell'assedio di Tournai venne effettuata una sorpresa dai nemici nell'area controllata dai Valloni, che erano "più spensierati che gl'altri".

131 Ibíd., f. 54v.

132 Ibíd., f. 44. 
erano meglio pagati in quanto dal loro paese venivano i finanziamenti della guerra $^{133}$ e ciò causava malumori fra valloni e italiani. Alessandro il 15 ottobre 1582 aveva ordinato che le sedici compagnie di fanteria italiana appena giunte fossero pagate come gli spagnoli e questi ultimi avevano violentemente protestato creando disordini ${ }^{134}$. L'ammutinamento dei valloni negli ultimi mesi del 1585 era stato dovuto alle loro proteste in quanto, come scrive Rinaldi, avevano

\begin{abstract}
"quelli di Spagna havute più paghe che loro", inoltre ritenevano che "tutti i buoni presidii, i gradi et le dignità si davano agli spagnoli, e a italiani, et che de fiamminghi et valloni non se ne teneva più conto, et che era la peggio pagata, rimunerata et maltrattata [nazione] di tutte"135.
\end{abstract}

Frédéric Perrenot lamentava nel suo memoriale al re che una compagnia di cavalleria italiana fosse pagata dodicimila fiorini al mese, e che il pagamento venisse fatto senza una rivista ("mostra") che avrebbe permesso di accertare il numero effettivo dei suoi membri: si faceva portavoce delle lamentele dei valloni che vedevano le truppe straniere trattate meglio di quelle del paese ${ }^{136}$.

Gravi problemi potevano derivare dalla scelta del comandante di unità multinazionali come rivela un passo di Coloma:

"Estava la Cavalleria ligera sin cabeça, por la enfermedad de Jorge Basta... y assi por no agraviar el Duque à ninguna de las tres naciones de que constava, nombrò tres cabos que la governasen à dias, cosa no vista hasta entonces, ni platicada despues. Por los Italianos se nombrò Apio Conti, por los Españoles Diego Davila Calderon y por los Albaneses Jorge Cresia"137.

Secondo Geoffrey Parker vi erano limitazioni nell' affidare il comando di contingenti nazionali e nell'aggregarsi ad essi: "Los españoles podian servir y mandar solo contingentes españoles" e esisteva una norma per cui era proibito a milanesi e a romani di prestar servizio in contingenti provenienti dal Regno di Napoli ${ }^{138}$. Seri disordini potevano, in effetti, verificarsi quando il comando di unità militari era affidato a uomini d'arme stranieri che non avevano con esse un buon rapporto; difficili erano in particolare, come scrive Rinaldi, le relazioni fra i Mansfelt e gli spagnoli:

133 Ibíd., f. 189: "... tutte le paghe venivano di Spagna e S. M.tà sempre comandava che se ne desse più agli spagnoli ch'agli altri, sendo essi de medesimi regni di dove venivano i denari". 134 Van der Essen, El ejercito, pp. 123 e 321; Esteban Estríngana, "Cabos de guerra", pp. 265293.

135 Rinaldi, Liber Relationum, f. 188v.

136 Mémoires de Champagney, pp. 274-275.

137 Coloma, Las guerras, pp. 166.

138 Geoffrey Parker, El ejército de Flandes y el Camino Español 1567-1659, (Madrid: Rialp, 1986), p. 65. 
"di natura sono altieri et non voglion esser comandati se non da gente di lor natione o da principi condottieri, et massimo havendo in odio il Conte [Mansfelt], il quale li aveva con molte male parole disgustati, svillanegiandoli stranamente e superbamente, capitani, e i maggior come i primi, non potendo celar la malevolenza che a lor portava"139.

Al ritorno delle truppe straniere nel 1582 Alessandro aveva tolto il comando della cavalleria spagnola a Giovan Battista Del Monte per assegnarla a Antonio De Olivera, militare di cui aveva una grande stima: alle proteste dell'italiano aveva risposto ricordandogli che non aveva mai trattato bene gli spagnoli e che quindi non poteva lamentarsi di averne perso la guida ${ }^{140}$.

\section{La reputazione della nazione italiana}

Per i militari delle varie nazioni era indispensabile mantenere buona la reputazione del loro contingente ed evitare che atti di viltà o indisciplina di singole unità danneggiassero l'immagine di tutti. Alessandro Farnese era particolarmente attento alla considerazione di cui godevano, presso la popolazione e presso i militari di altre nazioni, i suoi connazionali, di cui favoriva l'impiego ed a cui attribuiva, come vedremo, un ruolo determinante nella sua strategia militare.

Scriveva il ferrarese Gian Battista Montecatini nel gennaio 1583 al fratello Alberto che, a causa della resa di Megen compiuta dal capitano novarese Francesco Tornielli e della perdita della città e del castello di Eindhoven per colpa del capitano cremonese Girolamo Osio, gli italiani "avevano perso assai di reputazione per l'imprudenza et viltà mostrata"141. Nel 1581, nel corso dell'assedio di Tournai, un gentiluomo piemontese si era offerto di penetrare in un rivellino, ma l'impresa non ebbe successo: Rinaldi commenta che se lo avesse avuto, ne sarebbe derivato "honor di quel gentiluomo e della nazion italiana"142; a proposito della perdita di Breda nel 1590 il maggiordomo scrive:

"E gli spagnoli s'allegrarono e beffarono della nostra nazion italiana dicendo che eran già tre piazze che avevan perso per lor vigliaccheria, come era vero, ma questa era la più ignomignosa et più importante. $\mathrm{S}$. A. [Alessandro Farnese] la sentì oltra modo sì per il disservizio di S. M. come per disonore della sua nazione (in questo caso i militari coinvolti erano lombardi, toscani, umbri, siciliani)" ${ }^{\prime 143}$.

Nel commentare, infine, la morte del duca di Parma, Rinaldi scrive ancora che "la perdita di tant'uomo" rendeva tristi e faceva piangere "amaramente così per la cagion della fede come per l'interesse della riputazione del nome

\footnotetext{
139 Rinaldi, Liber Relationum, f. 190v.

140 Vázquez, Los sucesos de Flandes, I, pp. 369-370.

141 Cadoppi, "Un "macello di huomini da bene", pp. 158-159.

142 Rinaldi, Liber Relationum, ff. 95-95v.

143 Ibíd., f. 221.
} 
italiano"144. L'esigenza di preservare fra la popolazione una buona immagine dei militari italiani è espressamente dichiarata nel 1580 in una lettera del cavalier Andrea Cicogna a Cosimo Masi da Eindhoven: segnalava che a Tienen aveva notato circa quaranta archibugieri italiani a cavallo senza capo e sollecitava il segretario del generale a farne inviare uno al fine "di guardare I'honore della patria et evitar desordini", temendo, evidentemente, che azioni indisciplinate da parte loro potessero far danno alla reputazione dei soldati della sua nazione ${ }^{145}$.

L'ambasciatore veneziano Francesco Soranzo nella relazione sulla Spagna letta al Senato nel 1602 descriveva gli italiani nell'esercito "discordi per le proprie gare, facili a tumultuare per rispetti privati e non molto ordinati"146, e Pietro Fea nella biografia di Alessandro afferma che il generale era particolarmente disturbato dalle "risse che a quando a quando scoppiavano tra loro e si adoperava affinché non facessero "dir della nazione" e non dessero "ad intender alle altre nazioni che fra la nostra non c'[era] corrispondenza"147. Litigi fra italiani che potevano condurre a duelli, evitati solo per l'intervento dei superiori, sono frequentemente documentati nelle corrispondenze consultate. Nel 1582 Alessandro rimproverava il capitano Fabio Mataloni per una rissa avuta col capitano Moresino e minacciava di destituire entrambi se si fossero ripetuti "simili scandali"148. Nel 1583 il romano Biagio Capizucchi aveva avuto un contrasto con I'alfiere di Gravina e questo dava preoccupazione ad Alessandro che lo riprendeva duramente ${ }^{149}$. Nel marzo 1584 a Roermond vi fu l'incarcerazione effettuata da Fabio Mataloni del capitano Antonio Viganò che aveva presentato un cartello di sfida a Felice Fede $^{150}$. Nel 1590 in casa del conte Alessandro Rangoni a Bruxelles era scoppiata una lite fra il parmigiano Scipione Cavalca e il modenese Uguccione Rangoni che rischiò di concludersi con una sfida armata, se non fossero stati entrambi confinati nelle loro abitazioni ${ }^{151}$. Nel giugno 1592 in Francia a Chateau-Thierry vi fu un litigio fra Ranuccio Farnese ed il marchese del Vasto, cugino del duca di Mantova, che ebbe gravi conseguenze in Italia, suscitando perfino il timore che si giungesse ad uno scontro armato fra i due ducati padani $i^{152}$. Ranuccio era sospettato di aver avuto un ruolo nell'incendio dell'armeria dei Gonzaga e il marchese aveva mostrato di non ritenere del tutto infondate queste accuse: ne era seguito uno scambio di scritti che

\footnotetext{
144 Ibíd., f. 255.

145 ASP, CFE, 110, lettera di Andrea Cicogna a Cosimo Masi del 27 ottobre 1580 da Eindhoven. 146 "Relazione di Francesco Soranzo", in dirs. Nicolò Barozzi e Guglielmo Berchet, Relazioni degli stati europei lette al Senato dagli ambasciatori veneti nel secolo decimosettimo, Spagna, (Venezia: P. Naratovich, 1856), I, p. 131.

147 Fea, Alessandro Farnese, p. 475.

148 Ibíd., p. 475, n. 2, lettera di Alessandro Farnese a Fabio Mataloni del 6 ottobre 1582, in ASN, AF, 1659, distrutta.

149 Van der Essen, "Inventaire analytique" lettera di Alessandro Farnese a Biagio Capizucchi dal campo di Eeklo del 23 ottobre 1583, p. 76.

150 BNCR, Fondo Gesuitico, ms. 371, f. 121.

151 Cadoppi, "Un "macello di huomini da bene", pp. 167-169, lettere di Baldassarre Paolucci al duca di Ferrara del 24 gennaio e del 4 febbraio 1590.

152 Romano Canosa, I segreti dei Farnese, (Roma: Edizioni Multimediali, 2001), pp. 70-82.
} 
avevano circolato presso varie corti italiane e la Spagna ${ }^{153}$. Potevano nascere contrasti fra capitani italiani anche per i tentativi di alcuni di indurre militari di altre compagnie a passare nella propria ${ }^{154}$.

L'esercito di Alessandro veniva talvolta sconvolto da procedimenti giudiziari che si svolgevano negli stati della penisola: nel 1582 si era diffusa la voce che il conte Claudio Landi, accusato di congiurare contro Ottavio Farnese, avesse inserito quaranta suoi uomini nella fanteria italiana in Fiandra e tre di essi erano stati arrestati e condotti a Namur per essere interrogati e torturati dall'auditore militare, mentre quattro erano fuggiti calandosi dalle mura ${ }^{155}$. Da una lettera del luglio 1583 si apprende che a Lier nella compagnia di Odoardo Lanzavecchia vi era il già ricordato Girolamo Guazzotto, soldato quarantenne di Casalbagliano vicino ad Alessandria, che aveva preso parte alla guerra in Piemonte contro i francesi ed era al servizio di Muzio Landi da tre anni quando nel 1581 questi fu arrestato a Piacenza ${ }^{156}$. Interrogato dall'auditore militare Baldassarre de Ayala, Guazzotto aveva dichiarato di non essere mai stato informato della cospirazione a cui il suo padrone avrebbe preso parte e dopo essere stato a Rivalta, presso il conte Ottaviano Landi, fratello di Muzio, e a Felizzano nell'Alesssandrino presso un suo fratello, di essersi arruolato I'anno prima nel terzo in partenza per i Paesi Bassi: non aveva sottoscritto il documento del suo interrogatorio in quanto analfabeta ${ }^{157}$.

Anche il conte piacentino Ottavio Landi, gentiluomo della corte di Ferrara, che con raccomandazione di quel duca era venuto a combattere nei Paesi Bassi e a cui Alessandro aveva presto affidato incarichi di grande fiducia, era stato sospettato di essere connivente con il fratello Muzio condannato per la congiura contro il duca Ottavio ed era riparato a Parigi: si era sparsa, infatti, la voce che alcuni spagnoli per compiacere il generale intendessero eliminarlo e ne era stato informato da un teatino inviatogli dai capi militari spagnoli ${ }^{158}$.

Non mancavano anche rivalità fra italiani per cariche concesse da Alessandro Farnese: Coloma riferisce del dispiacere provato dal lombardo Ambrogio Landriani per la nomina del piacentino Pier Francesco Nicelli a capo della cavalleria nel 1591 avendo in precedenza ricoperta quella carica ed avendo una maggiore anzianità ${ }^{159}$.

\footnotetext{
153 Derks, "Le ricompense", pp. 205-230.

154 ASN, AF, 1699bis, lettera di Agabito Guadi a Cosimo Masi del 14 gennaio 1585 da Gand.

155 ASP, CFE, 111, lettera di Cosimo Masi ad Angelo Stirpio del 17 dicembre 1582.

156 ASP, CFE, 112, lettera di Cosimo Masi a Giovan Battista Pico del 4 luglio 1583 da Lier.

157 ASP, CFE, 112. La sua detenzione a Namur era durata quattro mesi, cfr. ASN, AF, 1699bis, lettera di Odoardo Lanzavecchia del 10 maggio 1585 da Lier.

158 Cadoppi, "Un "macello di huomini da bene", pp. 156-158.

159 Coloma, Las guerras, p. 126: "Y alli queriendo el Duque honrar a su Capitan de las guardias, Pedro Francisco Nicelli, le encargò el govierno de toda la Cavalleria, no sin disgusto de Don Ambrosio que le havia governado todo el año antes y era Capitan mas antiguo...".
} 


\section{Preferenza di Alessandro Farnese per la nazione italiana}

Alessandro, pur legato da stretti vincoli di parentela a Filippo II e nonostante l'educazione ricevuta alla corte spagnola, si considerava italiano ${ }^{160}$. Nel 1586 nella sua allocuzione ai militari italiani prima dell'assalto a Neuss aveva ricordato la "grandezza degli animi generosi" e il "valore", che aveva caratterizzato "sempre per ogni tempo e secolo la nostra nazione"161. Nei Paesi Bassi cercava di sottolineare agli occhi dei rappresentanti politici del paese le sue origini per rendere evidente la sua indipendenza dagli spagnoli: due dei suoi più importanti discorsi a loro diretti furono tenuti nella sua lingua, nelle trattative con i malcontenti nel 1578 e ed in quelle con i rappresentanti di Anversa per la resa della città nel $1585^{162}$. Alessandro aveva nel corso della sua conduzione delle operazioni militari chiaramente manifestato la sua preferenza per l'impiego di uomini d'arme italiani ed era stato per questo negli ultimi anni di vita duramente contestato dai militari iberici ${ }^{163}$. Secondo Coloma la sua propensione ad un maggior impiego degli italiani si era manifestata solo dopo la morte del duca Ottavio Farnese, il quale aveva ricordato spesso al figlio che il vantaggio derivante dalla competizione nel combattere fra le due nazioni sarebbe stato più che compensato dal danno derivante dalla gelosia che gli spagnoli avrebbero nutrito verso i suoi compatrioti ${ }^{164}$. Contestata fu la scelta di Alessandro Farnese di affidare nel 1585, dopo la morte del marchese di Roubaix, il comando della cavalleria leggera al marchese del Vasto (nomina confermata I'anno seguente da Filippo II), che deluse particolarmente le aspettative dei capi valloni ${ }^{165}$. Criticata fu anche la sua decisione di nominare Odoardo Lanzavecchia governatore di Gertruidenburg oltre che di Breda ${ }^{166}$.

\footnotetext{
160 Per i rapporti di Alessandro Farnese con Filippo II, cfr. José Eloy Hortal Muñoz, Los asuntos de Flandes. Las relaciones entre las Cortes de la Monarquía Hispana y de los Países Bajos durante el siglo XVI, (Saarbrücken: Editorial Académica Española, 2011), cap. 5; María José Rodríguez-Salgado, "Kinship, collaboration and conflict: the complex relationship between Alessandro Farnese and Philip II", in En la Corte del Rey de España. Liber Amicorum en homenaje a Carlos Gómez-Centurión Jiménez (1958-2011), dirs. Rafael Valladares, Feliciano Barrios e Juan Antonio Sánchez-Belén, (Madrid: Polifemo, 2016), pp. 61-105.

161 Rinaldi, Liber Relationum, f. 198.

162 Ibíd., f. $183 \mathrm{v}$.

163 Giuseppe Bertini, "Alessandro Farnese fra Italia, Spagna e Paesi Bassi", in Cheiron, XXVII, (2010), pp. 71-95. Per il risentimento spagnolo nei riguardi di Alessandro Farnese a causa del trattamento da lui riservato ai militari della loro nazione, cfr. il discorso di Hernando de Toledo al Consiglio di Stato, in Luis Cabrera de Cordoba, Felipe Segundo, (Madrid: Sociedad de Bibliófilos Españoles, 1877), IV, p. 326.

164 Coloma, Las guerras, p. 67; cfr. Bertini, Alessandro Farnese fra Italia, p. 85.

165 Rinaldi, Liber Relationum, ff. 175v-176: "Per la morte di Roubais venne a mancare I'ufficio del Generalato della Cavalleria, che sebbene molti del paese pretendevano quel grado, il Principe lo conferì per trarlo inanzi et fargli onore al Marchese giovanetto di Pescara, per modo di provisione tanto che venisse la conferma dal Re, con gran disgusto del Marchese di Arentin e del Principe di Simay cavalieri principali di questi Stati, che ciascun pretendeva come grado che s'avesse da conferire a cavalieri principali del paese. Venne la riconferma del Re con gran scontento di tutta questa nobiltà, che con buone parole Parma acquietò tutti. Che col tempo il Guasto messe resia (?) fra il Duca di Mantova e il Principe di Parma, figliol d'Alessandro, dimostrando di questo favor ricevuto dal Farnese non molta gratitudine a S. A. et alla sua casa".

166 Coloma, Las guerras, p. 64.
} 
La preferenza di Alessandro per gli italiani è rappresentata da Rinaldi come diretta conseguenza dell'avversione della popolazione verso gli spagnoli che l'azione del duca d'Alba aveva suscitato nel paese. Scrive, infatti, che un grande successo del governatore era stato quello di ottenere nel 1582 dai capi delle province riconciliate il consenso al richiamo di soldati spagnoli:

"che questo non si saria mai creduto, et con ogni gran ragione, sendo stati offesi da loro nella roba, nel sangue et nell'onore e patito da loro ogni infortunio, oltre l'odio ordinario et comune che queste genti hanno con quella nazione, certo questa è stata una gran gloria d'Alessandro d'avere riconciliato due nazioni lontane et avverse, di paesi e di costumi, alienate l'una dall'altra per acerbissimi odii particolari, universali per inimicizie state et durate molti anni"167.

La scelta di impiegare italiani è, implicitamente, considerata da Rinaldi inevitabile per Alessandro: se voleva riportare il paese sotto il controllo del re e della chiesa cattolica, doveva utilizzare uomini d'arme provenienti dalla penisola, in quanto fedeli alla religione romana, non ostili alla Spagna, ma allo stesso tempo non invisi alla popolazione locale, di cui era indispensabile ottenere la collaborazione per la conduzione della guerra. Come giustamente sostenuto da Sebastiaan Derks, il maggiordomo voleva con la sua opera rispondere alle gravi accuse che erano state rivolte ad Alessandro da parte spagnola168: fra queste, la maggiore era quella di aver concesso un trattamento preferenziale agli italiani e di aver fortemente penalizzato i militari iberici. L'opinione di Rinaldi (e di Alessandro) che gli italiani fossero meglio accetti degli spagnoli dalla popolazione non era, tuttavia, da tutti condivisa, come si coglie dal memoriale di Frédéric Perrenot, il quale definisce i militari provenienti dalla penisola "plus déréglés que ne l'ont jamais été les Espagnols et que I'on n'a pas moins envie de s'en débarasser que des Espagnols eux-mêmes"169.

\section{Guarnigioni italiane di città fiamminghe.}

Capitani e compagnie italiane nell'esercito di Alessandro Farnese potevano essere destinate a combattere nelle annuali campagne per conquistare nuovo territorio o per difendere quello acquistato o, alternativamente, a risiedere di guarnigione in forti $O$ in città fiamminghe. Come scriveva nel 1584 I'ambasciatore veneziano Matteo Zane, il generale avrebbe avuto bisogno di ventimila uomini sul campo, ma non riusciva ad averne più di diecimila perché doveva "lasciar le piazze ben presidiate rispetto agl'inimici di fuori e ai popoli di dentro"170. I presidi, resi necessari dalla vicinanza di posizioni nemiche e dalla presenza di cittadini non cattolici, pronti ad aprire le porte alle truppe

167 Rinaldi, Liber Relationum, f. 109.

168 Derks, "Le ricompense", pp. 205-230.

169 Mémoires de Champagney, cit., p. 308.

170 Relazione di Matteo Zani, p. 347. 
degli stati ribelli, erano spesso rifiutati dalle città con grave rischio per la loro difesa in caso di assedio: militari stranieri all'interno delle mura comportavano notevoli aggravi per il mantenimento, imposto spesso dai trattati di resa, e turbative alla quiete e particolarmente sgradite erano le guarnigione spagnole per il ricordo di atrocità commesse in un passato recente (alcune città nelle loro condizioni di resa si riservavano il diritto di scegliere la nazionalità delle truppe della guarnigione) ${ }^{171}$. Quando cessava il pericolo veniva tolto il presidio e come scriveva Andrea Cicogna da Lovanio nell'agosto 1585 ai cittadini "pare[va] di stare in paradiso senza guarnigione"172.

Alessandro, come scrive Rinaldi, aveva nominato governatori spagnoli ad Anversa, Gand, Sas van Gent, Dunquerke, Sluis, alcuni fra i maggiori centri del paese, ed aveva scontentato valloni e fiamminghi che detestavano quella nazione e avrebbero ambito a questi incarichi: se, tuttavia, "havesse messo in queste piazze gente del paese, alla morte sua haverien forse accordatosi o tutti o parte con il nemico e fatto peggio che mai"173. La scelta di nominare governatori e guarnigioni italiane era quindi obbligata come ci lascia intendere il maggiordomo: avevano, infatti, governatori italiani Breda, Lier, Nimega, Roermond e Vilvoorde, assegnate rispettivamente a Camillo Del Monte, Mario Cardoino, Nicolò Cesis, Andrea Cicogna e Ambrogio Landriani, uomini d'arme con buona conoscenza del paese, con ottima reputazione già acquisita sul campo di battaglia, disposti a ricoprire una posizione più tranquilla, anche se giudicata da Alessandro meno "onorevole" della partecipazione alle campagne militari da lui annualmente intraprese ${ }^{174}$. Molti erano i centri urbani con guarnigioni di compagnie italiane o con di altre nazioni con capitani italiani, che coesistevano con governatori di altre nazioni: Aarschot (Antonio Cavaccio), Alost (Giorgio Masnieri), Lier (Mario Cardoino), Lovanio (Fabio Mataloni), Maastricht (Ercole Ricci), Medelar (Camillo Sacchini), Namur (Ippolito Maria Rossi), Roermonde (Ruggero Veronici), Tienen (Fracchia), Tournai (Matteo Corvini), Weert (Camillo Del Monte, Appio Conti); a Halle, eccezionalmente, era stata lo stesso borgomastro che aveva chiesto a Francesco Biumi (Corradino) di insediarvisi con i suoi uomini e Alessandro Farnese gli aveva allora concesso la patente di governatore ${ }^{175}$.

Nelle corrispondenze esaminate vi sono frequenti riferimenti ai difficili rapporti intrattenuti da vari capitani italiani con le autorità locali. Ercole Rizzi, a capo di una compagnia di fanti lussemburghesi, denunciava nel 1580 a Cosimo Masi "il maltrattamento" dei suoi soldati di guarnigione a Maastricht, "pieni di debiti, nudi et senza aver alcuno avantaggio che si usa[va] in tutti

\footnotetext{
171 Joseph Lefèvre, Correspondence de Philippe II sur les affaires des Pays-Bas, (Bruxelles: Palais des Academies, 1953), II, p. 416, lettera di Alessandro Farnese al re del 26 settembre 1583.

172 ASN, AF, 1699bis, lettera di Andrea Cicogna ad Alessandro Farnese dell'8 agosto 1585 da Lovanio.

${ }^{173}$ Rinaldi, Liber Relationum, f. 253. Per le cariche di governatori e castellani, cfr. González de León, The Road to Rocroi, pp 27-29.

174 Rinaldi, Liber Relationum, f. 252v.

175 Narrativa di quanto ha fatto Francisco Biumio detto Corradino..., in Bertini, Militari italiani, p. 246.
} 
I'altri luochi" (non riceveva dalla città sussidi per sè e per i cavalli). I soldati non stavano volentieri in quella città perché non era loro permesso "sgrafignar niente", ma ugualmente egli era riuscito a farsi benvolere da loro grazie alla sua generosità ed allo stesso tempo ad incontrare il favore dei cittadini perché non tollerava da parte dei suoi uomini comportamenti scorretti. La situazione era resa difficile dal governatore che non accettava militari di "natione estrangiera": sarebbe stato difficile a lungo andare evitare che i suoi uomini facessero "tumulti" e desiderava, pertanto, essere trasferito in una città dove sperava di poter finalmente trattenere per sé la sua paga (eventualmente a Weert, il cui governatore voleva liberarsi di un capitano italiano di nome Camillo che non controllava i suoi soldati) ${ }^{176}$.

In una lettera indirizzata nel 1580 da Roermond a Cosimo Masi il capitano Ruggero Veronici di Todi segnalava I'ostilità del governatore della città per le due compagnie d'italiani, "belle piene et di buoni soldati", che vi si trovavano di guarnigione: ne auspicava la partenza, "non obstante che veramente vivano con più obbedienza et modestia che nessuno delli altri" e il capitano umbro sollecitava la concessione di una compagnia di valloni, che avrebbe messo "in piedi dentro di ventiquattro giorni et di buoni soldati" con l'aiuto che il governatore gli aveva promesso ${ }^{177}$.

Ottavio Tarlantini comunicava ad Alessandro da Breda nel 1589 che "li borgomastri delli villaggi che ci [erano] stati consegnati dal Cav. Cicogna" [commissario dei viveri] avevano fatto ricorso e non intendevano "pagare cosa nissuna", per cui per mantenere i suoi soldati aveva dovuto indebitarsi con gli amici ${ }^{178}$. Pagamenti in danaro venivano corrisposti alle guarnigioni con i proventi dei dazi fluviali affidati in monopolio a Paolo Rinaldi ${ }^{179}$ : Frédéric Perrenot avanzava forti sospetti sulla onestà dell'italiano e incolpava questi dazi di essere la causa dell'emigrazione della popolazione e dell' impoverimento del paese ${ }^{180}$.

Rinaldi scrive che per manifestare la sua benevolenza ai cittadini di Deventer, con i quali aveva raggiunto un accordo dopo che si erano sottratti al controllo del conte di Leicester, il generale aveva messo il "presidio in una parte che non dava alla città né alle persone impaccio alcuno"181. Secondo Violet Soen, egli aveva imposto in molte città la ricostruzione di cittadelle al fine di evitare che i soldati fossero alloggiati presso i cittadini e causassero

\footnotetext{
176 ASP, CFE, 110, lettere di Ercole Ricci a Cosimo Masi del 9 e 21 settembre 1580 da Maastricht.

177 ASP, CFE, 110, lettera di Ruggero Veronici a Cosimo Masi del 25 agosto 1580 da Roermond. 178 ASN, AF, 1722 bis, lettera di Ottavio Tarlantini ad Alessandro Farnese del 2 maggio 1589 da Breda.

179 Lefèvre, Correspondence di Philippe II, II, p. 615, lettera di Alessandro al re del 20 dicembre 1591: "Les licenses perçues sur la Meuse aideront au paiement des garnisons établies dans les diverses villes".

180 Mèmoires de Champagney, pp. 266-267.

181 Rinaldi, Liber Relationum, f. 205.
} 
loro disagi ${ }^{182}$. La minuta di una lettera di Alessandro ad Ambrogio Landriani del 1588 ci illumina sulla sua strategia nei riguardi degli abitanti delle città:

"[Ho] appena finito d'intendere come per ordine suo certi soldati della sua compagnia hanno essecutato la villa di Tillemont per conto delle contribuzioni che pagano a detta compagnia levandoli li bestiami et usandoli altri termini rigorosi e poco decenti, in sopra viene avviso del magistrato di Brusseles che altri soldati suoi et pur di suo ordine sono entrati in certa casa del burghemastro di detta villa, situata a Gaesbeche, et hanno rotto le porte et cofani et levatone biancherie et altre robe et portatosele et menatose un cavallo sotto pretesto di certo residuo che lei pretende da essa villa per causa della ricuperatione di Malines, et sebene il primo avviso mi causasse il dispiacere che di sì strano procedere lei stessa si possa immaginare, nondimeno quest'altro $\mathrm{mi}$ ha ... scandalizzato[per]l'inconveniente che simil modo di procedere ha causato et suol causare negli animi del comune popolo... Così al burghemastro come a quei di Tillemont sia restituito quello che se li è tolto fino a un pelo"183.

Ugualmente in una lettera del 19 febbraio 1585 di Alessandro a Biagio Capizucchi, con la sua compagnia al presidio di Weert, raccomandava la disciplina per evitare che i borghesi abbandonassero la città ${ }^{184}$.

\section{Alessandro Farnese e gli abitanti delle campagne}

Il comportamento che Alessandro imponeva alle sue truppe nei riguardi della popolazione delle campagne è descritto in un passo di Rinaldi: il generale faceva "haver sempre riguardo a poveri villani" e teneva "a freno i soldati con speranza all'occasione di ricevere da queste genti aiuti e servitii a bisogni del campo"; cercava di fare in modo che i villaggi "non fossero deserti, né che i soldati facessero danno a loro fuor d'uso o di misura". Non era facile, tuttavia, conquistarsi la fiducia dei contadini "per esser loro tanto usi ad esser malamente trattati", per cui "per tema de nostri abbandonavano ... tutte le case" di ricchi villaggi che erano soliti mantenere in precedenza gran parte dell'esercito nemico ${ }^{185}$. Lo stesso concetto è espresso in un altro passo del maggiordomo:

182 Violet Soen, "Reconquista and Reconciliation in the Dutch Revolt: The Campaign of Governor General Alessandro Farnese (1578-1592)", in Journal of Early Modern History, XVI, (2012), pp. 1-22.

${ }_{183}$ ASN, 1722 bis, minuta di lettera di Alessandro Farnese ad Ambrogio Landriani del 15 luglio 1588 da Bruges.

184 Van der Essen, "Inventaire analytique".

185 Rinaldi, Liber Relationum, f.114. Così proseguiva: "Con tutto ciò dispiaceva al Principe fuor di modo queste insolenze de soldati et miserie di questi uomini, sendosi sempre egli governato con dolcezza con tutti, parendogli che la piacevolezza col dolce governo porta maggior utile che il governar aspramente non già che gli paresse come diceva lui che s'abbia d'aver rispetto agl'inimici, se non circa a un certo che, o ai soldati che mal si portino in pregiudizio della giustizia che al grido di questo nome benigno". 
"Trovavasi Parma tutti i suoi soldati molto scorretti et indisciplinati...e tanto licenziosi et temerari che n'haveva ogni di mille volte richiami, dandosi alle rapine e ad ogni sorte di violenze, per la qual cosa deliberò ridurgli in buona disciplina militare et in obbedienza, vedendo che gli Alamanni venuti nuovamente pigliavano anche loro di questi cattivi usi, fece chiamar tutti i colonnelli, capitani et officiali e cominciò con amore a riprendere tutti con dir che questo non era il vero esercizio del soldato, ma sebbene officio d'insolente, frabuttero ${ }^{186}$ et assasin di strada e che l'honor e il valore degli eserciti di milizia perfetta non sono fondati in sul rubare e usar violenza e forza ai poveri villani, ma in sul vincere i nemici e acquistar honore et fama, non potendo durar compagnia d'huomini se non sono raffrenati gli scorretti e puniti i cattivi..."187.

Particolarmente significativa, infine è la relazione del capitano novarese Francesco Biumi, detto Corradino, in cui si presentava come fedele esecutore degli ordini di Alessandro e sottolineava più volte come avesse sempre cercato di ottenere il favore della popolazione rurale da cui riceveva in cambio informazioni utili nella lotta contro il nemico ${ }^{188}$ : in tre occasioni fra il 1580 ed il 1584 si era messo all'inseguimento delle truppe nemiche che avevano saccheggiato i beni dei contadini e aveva loro restituito quanto era stato sottratto ${ }^{189}$.

Il malcontento ed il disagio dei soldati era in parte causato, secondo Rinaldi, dall'avarizia dei capi che privando i loro uomini di mezzi di sostentamento li costringevano a procurarseli con la violenza sulla popolazione: il conte Carlo Mansfelt era da lui accusato di appropriarsi di quanto raccolto nei villaggi che avrebbe dovuto, invece, essere destinato ai soldati ${ }^{190}$ e di taglieggiare piccoli centri rurali pur "amici e parziali del Re, facendogli pagare l'alloggiamento a denari"191. I due eserciti nemici si contendevano il controllo della campagna da cui si procuravano viveri per gli uomini d'arme ${ }^{192}$ e l'approvvigionamento

\footnotetext{
186 Farabutto.

187 Rinaldi, Liber Relationum, f. 92v-93. Così proseguiva: "...facendo intendere a tutti $i$ colonnelli, capitani, alfieri, sergenti et officiali et con comandamento a stretto nodo a pena della disgrazia sua che facessero star ne termini tutti i soldati che militavano sotto il suo stendardo e che gli facessero osservar gl'ordini e sopra tutto esser prontissimi a eseguirli facendogli lasciare et dimenticare tanta avarizia e licenza, che s'havevano presa, notificando loro che chi non obbediva da qui innanzi patiria la pena del presente delitto e del passato, rappresentando il male di quel che è cagione e la virtù per il contrario quanta reputazione e fama ci reca con la grazia di Dio e degli huomini et che egli era disposto in tutte l'opere eccellenti participar con loro d'ogni travaglio e fatica il di et la notte, con questo, che tutti i premi che si conseguiranno fossero tutti loro e de soldati, com egli haveva fatto sempre".

188 Narrativa di quanto ha fatto Francisco Biumio detto Corradino..., in Bertini, Militari italiani, pp. 243-261.

${ }_{189}$ Ibíd.

190 Rinaldi, Liber Relationum, f. 190v: Mansfelt "tirava dalle terre e villaggi convicini più di quindicimila fiorini il mese... per la più parte se li salvava per uso suo... ".

191 Ibíd., f. 218.

192 Ibíd., f. 218: con l'acquisto di Gertruidenberg "tutti i villaggi vicini d'Olanda soggetti ai nemici furono portati a contribuire ai Reali, oltre che di questi luoghi si cavava grande entrate
} 
dell'esercito, ottenuto grazie ai buoni rapporti con gli abitanti dei villaggi, è secondo Rinaldi una delle costanti preoccupazioni di Alessandro ${ }^{193}$.

\section{La disciplina nell'esercito di Alessandro Farnese}

\section{Scrive Rinaldi che Alessandro}

"in tutti i luoghi ebbe una corte grandissima di diverse qualità di persone e di più nazioni et senza i pedoni et vivandieri et altri che seguivano il campo alloggiavano i furieri in campagna più di mille e cinquecento cavalli, tutti ridotti a buono et quieto vivere senza malignità nessuna che parevano proprio dal maggiore al minore una compagnia d'uomini da bene, pacifici et come amorevoli fratelli"194.

Per ottenere questo risultato doveva essere necessaria una rigida applicazione di regole di comportamento, le stesse che dovevano essere imposte, oltre che agli uomini della sua corte, ai militari nel corso delle operazioni di guerra e a quelli di presidio nelle città. La disciplina era ciò che Alessandro richiedeva ai suoi uomini e a questo fine aveva adottato nel 1587 un codice da osservare nei tribunali cui venivano deferiti i militari in caso di infrazioni ${ }^{195}$. Un eminente giurista spagnolo, laureato all'Università di Lovanio e autore di due trattati di diritto, Baltasar de Ayala, era stato nominato nel 1580 dal re, su sua raccomandazione, auditore militare e ricoprì la carica fino alla morte nel $1584^{196}$. Inoltre, per evitare disagi alla popolazione costretta ad ospitare i militari nelle loro abitazioni e per eliminare motivi di contesa, Alessandro emanava disposizioni elencando quello che i soldati avrebbero potuto richiedere ai padroni delle case in cui alloggiavano: fra le carte dei Gesuiti romani si trova, firmato dal generale il 4 febbraio 1590 a Bruxelles, I"Ordine et forma da tenersi nell'alloggiamento del Terzo d'Infanteria del Colonnello Camillo Capizucchi che va ad inverno nel Borgo di S. Amando"197. Un analogo documento in cui si specifica il comportamento dei soldati della guarnigione verso gli abitanti di Nieuwpoort è contenuto in un codice della Biblioteca Vaticana, consultato da Van der Essen ${ }^{198}$.

In un inedito trattato coevo sull'arte militare conservato nell'Archivio di Stato di Parma si ha una descrizione di come un soldato avrebbe dovuto evitare l'ozio, causa di disordini:

di pescagioni, sia di storioni, salmoni, lucci, tutto pesce eccellentissimo delli quali se ne insala un numero senza fine che ne va per ogni parte del mondo".

193 Per l'importanza dell'approvvigionamento per la conduzione delle operazioni militari, cfr. Giovanni Muto, "Apparati militari e fabbisogno finanziario: il caso della Spagna "de los Austrias", in Donatie et Kroener, Militari e società civile, pp. 23-52.

194 Rinaldi, Liber Relationum, f. 253.

195 Ángel Riesco Terrero, "La ordenanza e instruccion del General Farnesio (Bruselas 1587)", in El ejército y la armada de Felipe II ante el IV centenario de su muerte, (Madrid: Ministerio de Defensa, 1997), pp. 71-76; González de León, The Road to Rocroi, pp. 113-116.

196 Ved. sopra, I'interrogatorio da parte di Ayala di Girolamo Guazzotto.

197 BNCR, Fondo Gesuitico, ms. 371, f. 109.

198 Van der Essen, El ejército, p. 119. 
"...si chè qualora sia disoccupato i suoi passatempi saranno il giocar d'arme, cavalcare, giocar alla palla, tirare il palo, correre, saltare, e simili, i quali esercizi e più agile e più sano lo conserveranno. Dovrebbe talora pascer l'anima col legger libri virtuosi e particolarmente quelli che trattano del suo mestiere, o veramente storie dalle quali ne caverà documenti di ragione, di Stato e di guerra..."199.

Nel descrivere la formazione di Alessandro in età giovanile e i passatempi da lui praticati in età adulta, Rinaldi ci fa conoscere che l'attività fisica occupava molte ore della sua giornata ed il trattato sembra ispirato al suo stile di vita. Nell'esercito del generale era praticato, fra l'altro, nel tempo libero il gioco del "calcio nel prato": alcuni venturieri e capitani italiani chiedevano che fosse autorizzato per i loro uomini e dettavano le regole per partite da giocarsi nei giorni di carnevale ${ }^{200}$. Alessandro non amava il gioco d'azzardo e per entrare nelle sue grazie era preferibile non esservi dedito ${ }^{201}$ : al giovane milanese Federico Cusani, assunto nella sua casa su raccomandazione del duca Ottavio, fu imposto di giurare che si sarebbe astenuto dal gioco, vizio in cui era evidentemente caduto ${ }^{202}$. I debiti da onorare avrebbero potuto essere causa di ruberie ai danni della popolazione e quindi fonte di indisciplina, per cui non era gradito nell'esercito al tempo del generale italiano. Anche presso militari di altre nazioni era diffuso il gioco d'azzardo, come documentano le raccomandazioni di Juan Idiáquez al figlio ${ }^{203}$ e le disposizioni per i soldati degli stati ribelli citate da Erik Swart ${ }^{204}$.

Comportamenti indisciplinati dettati dall'avidità di denaro potevano portare al fallimento di importanti operazioni militari o a situazioni molto rischiose: nel testo di Rinaldi si descrive come a Ostenda nel 1585 il saccheggio da parte dei militari reali che vi erano penetrati sotto la guida di un capitano vallone diede opportunità ai nemici di riprendere il controllo della città ${ }^{205}$ e come nello stesso anno i soldati spagnoli, attratti dalla possibilità di depredare con facilità ricchi villaggi sull'isola di Bommel, vi sarebbero stati sopraffatti dalle truppe nemiche se non fosse intervenuta una provvidenziale gelata delle acque ${ }^{206}$.

\footnotetext{
199 ASP, Fondo manoscritti, 59, Trattato d'arte militare, f. 2v.

200 BNCR, Fondo Gesuitico, ms. 371, f. 96. I capitani che avevano fatto I'appello erano: Agostino Arconati, Lodovico Gambaloita, Nicolò Rangoni, Alessandro Torelli, Matteo Salati, Giovan Battista Fioravanti, Pietro Maria Balestrazzi, Mandricardo Bresciani, Alfonsi Tassi, Antenore Billi, Nicolò Rossi, Giovan Antonio Abonenti (questi sono i nomi che nel documento, alquanto deteriorato, si possono leggere).

201 Instruttione all'Illustrissi. Signor Pietro Caietano quando andò in Fiandra, in La seconda parte del Thesoro Politico, (Milano: Bordone, 1601), pp. 502-517: "Non gioca Sua Altezza né a dadi, né a carte, et è a credere che coloro che le sono più simili, debbiano conseguentemente essergli più in gratia".

202 ASP, CFE, 113, lettera di Paolo Rinaldi a Papirio Picedi del 13 luglio 1585.

203 Cfr. lettera conservata presso la Biblioteca Casanatese di Roma, citata in Leon Van der Essen, "Croisade contre les hérétiques ou guerre contre des rebelles", in Revue d'histoire ecclésiastique, LI, (1956), pp. 42-78.

204 Erik Swart, "From "Landsknecht" to "Soldier": The Low German foot soldiers of the Low Countries in the second half of the Sixteenth Century", in International Review of Social History, LI, (2006), pp. 75-92.

205 Rinaldi, Liber Relationum, ff. 169v-170v.

206 Ibíd., ff. 189v-190.
} 
La mancanza di disciplina di una guarnigione italiana sotto il comando del governatore Monsignor di Benthy ne aveva determinato la cacciata da Venlo e la conseguente perdita della città nel 1590: "I cittadini non potendo sopportare i cattivi portamenti de' soldati, che per ordinario sono audaci et impertinenti, e nelle guarnigioni si mostrano più che altrove libidinosi, avari et insaziabili, li scacciarono dalla terra..."207.

\section{Conclusioni}

Le ricerche sulla composizione del contingente italiano nell'esercito di Alessandro Farnese e sulla strategia del generale nel condurre la guerra, evidenziata dal testo di Rinaldi e dalle lettere scambiate con i suoi capitani, permettono di giungere alla conclusione che la sua preferenza all'impiego di militari della nazione italiana non fosse esclusivamente determinata dal perseguimento di interessi dinastici o da eccessivo e cieco nazionalismo. Gli uomini d'arme del suo paese potevano assicurargli, infatti, una migliore esecuzione degli obiettivi militari che si prefiggeva: grazie alle parentele che lo legavano ad alcuni di essi, ai rapporti che intratteneva con i sovrani degli stati di cui erano originari, all'appartenenza di altri a famiglie di servitori della sua casa e di altri ancora a famiglie feudali del suo stato, il generale era in grado di imporre loro una più rigida disciplina e di controllarne le azioni con più efficacia di quanto avrebbe fatto con militari di altre nazionalità. Un buon rapporto con gli abitanti delle città presidiate e con coloro che risiedevano nelle campagne era da lui ritenuto indispensabile per ottenere collaborazione nelle operazioni militari volte al controllo del territorio e un flusso costante di prodotti agricoli necessari al sostentamento delle truppe. L'impiego preferenziale dei suoi connazionali, tuttavia, come si è visto, espose il generale ai sospetti degli spagnoli e determinò la diffusione fra di essi di un profondo malcontento, che indusse il re, in fine, a deciderne la destituzione.

L'importante ruolo riservato da Alessandro agli italiani nella guerra dei Paesi Bassi e l'acquisizione da parte loro di conoscenze in campo militare ebbero un effetto positivo per l'Italia. L'esperienza acquisita in Fiandra fra il 1578 ed il 1592 fu ampiamente utilizzata da Venezia nella sua lotta contro i turchi: alcuni dei documenti rintracciati nel corso della ricerca dimostrano come i veneziani fossero attenti e tempestivi nel cogliere l'opportunità di avvalersi sul fronte orientale di italiani formati alla "scuola" del generale. Lo stesso si può dire della chiesa romana che inviò veterani della guerra nei Paesi Bassi a sostenere lo sforzo delle truppe imperiali in Ungheria e di quelle della Lega in Francia. Anche la Spagna trasse beneficio dalla formazione impartita da Alessandro Farnese ai suoi connazionali: nel corso del Seicento, infatti, la monarchia asburgica affidò in gran parte la difesa dei suoi interessi nei Paesi Bassi e in Germania a uomini d'arme provenienti dalla penisola, riconoscendone il valore e apprezzandone le conoscenze dell'arte militare.

207 Campana, Delle historie del mondo, II, p. 448. 
Bibliografía:

Adami 1685: Annibale Adami, Elogi storici de' due Marchesi Capizucchi fratelli Camillo e Biagio celebri guerrieri del secolo passato, (Roma: Stamperia della Reverenda Camera Apostolica, 1685)

Adriaenssen 2008: Leo Adriaenssen, Staatsvormend geweld. Overleven aan de frontlinies in de meierij van den Bosch 1572-1629, (Tilburg: Stichting Zuidelijk historisch contact, 2008)

Arfaioli 2011: Maurizio Arfaioli, "Giovan Battista Marino e gli "Alfonsi d'Avalos", in Studi secenteschi, LII, (2011), pp. 405-411.

Bertini 2007: Giuseppe Bertini, Ottavio Gonzaga di Guastalla. La carriera di un cadetto al servizio della monarchia spagnola (1543-1583), (Guastalla: Biblioteca Maldoti, 2007)

Bertini 2010: Giuseppe Bertini, "Alessandro Farnese fra Italia, Spagna e Paesi Bassi", in Cheiron, XXVII, (2010), pp. 71-95.

Bertini 2013: Giuseppe Bertini, "Carriere di militari italiani nell'esercito di Alessandro Farnese nei Paesi Bassi: merito o privilegio", in Militari italiani dell'esercito di Alessandro Farnese nelle Fiandra, dir. Giuseppe Bertini, (Fidenza: Mattioli, 2013), pp. 171-203.

Brom 1908-1914: Gisbert Brom, Archivalia in Italie belangrijk voor de geschiedenis van Nederland, ('S-Gravenhage: M. Nijhoff, 1908-1914), 3 vols.

Brunelli 1997: Gianpiero Brunelli, "Soldati della scuola vecchia di Fiandra". Nobiltà ed esercizio delle armi nello Stato della Chiesa fra Cinque e Seicento", in I Farnese. Corti, guerra e nobiltà in antico regime, dirs. Antonella Bilotto, Piero Del Negro, Cesare Mozzarelli, (Roma: Bulzoni, 1997), pp. 421-444.

Brunelli 2003: Gianpiero Brunelli, Soldati del papa. Politica militare e nobiltà nello Stato della Chiesa (1560-1644), (Roma: Carocci, 2003)

Brunelli 2013: Gianpiero Brunelli, "Con insonnij diversi, et visioni stravaganti di guerre": I'esordio di Pietro Caetani nelle Fiandre", in Militari italiani dell'esercito di Alessandro Farnese nelle Fiandra, dir. Giuseppe Bertini, (Fidenza: Mattioli, 2013), pp. 75-106.

Cabrera de Córdoba 1877: Luis Cabrera de Cordoba, Felipe Segundo, (Madrid: Sociedad de Bibliófilos Españoles, 1877), 4 vols.

Cadoppi 2013: Alberto Cadoppi, "Un "macello di huomini da bene". Lettere al duca d'Este dalle Fiandre di militari al servizio di Alessandro Farnese", in 
Militari italiani dell'esercito di Alessandro Farnese nelle Fiandra, dir. Giuseppe Bertini, (Fidenza: Mattioli, 2013), pp. 153-170.

Caetano 1601: Instruttione all'Illustrissi. Signor Pietro Caietano quando andò in Fiandra, in La seconda parte del Thesoro Politico, (Milano: Bordone, 1601), pp. 502-517.

Campana 1607: Cesare Campana, Delle historie del mondo, II (Venezia: I Giunti, 1607)

Canosa 2001: Romano Canosa, I segreti dei Farnese (Roma: Edizioni Multimediali, 2001)

Coloma 1625: Carlos Coloma, Las guerras de los Estados Baxos desde el año de MDLXXXVIII hasta el de MDXCIX, (Amberes: Casa de Pedro y Iuan Bellero, 1625)

De Groof 1997: Bart de Groof, "Una questione di priorità: spagnoli, italiani e fiamminghi alla morte di Alessandro Farnese", in I Farnese. Corti, guerra e nobiltà in antico regime, dirs. Antonella Bilotto, Piero Del Negro, Cesare Mozzarelli, (Roma: Bulzoni, 1997), pp. 167-187.

De Robaulx de Soumoy 1860: Aimé Louis Philemon De Robaulx De Soumoy (dir.), Mémoires de Champagney. Discours sur les affaires des Pays-Bas, 1573-1590, avec notice et annotations, (Bruxelles: Société de I'Histoire de Belgique, 1860)

Derks 2013: Sebastiaan Derks, "Le ricompense della guerra: giustificazione e rappresentazione di Alessandro Farnese nel "Liber relationum" di Paolo Rinaldi", in Militari italiani dell'esercito di Alessandro Farnese nelle Fiandra, dir. Giuseppe Bertini, (Fidenza: Mattioli, 2013), pp. 205-230.

Derks 2015: Sebastiaan Derks, "The fruits of war: the representation of Alessandro Farnese in Paolo Rinaldi's Liber Relationum", in Dynastic Identity in Early Modern Eyrope, dirs. Liesbeth Geevers, Mirella Marini, (Farnham: Ashgate, 2015), pp. 153-178.

Essen 1926: Léon van der Essen, Les Italiens en Flandre au XVI et au XVII siècle, (Bruxelles: La Lecture au Foyer, 1926)

Essen 1931: Léon van der Essen, "Inventaire analytique d'un recueil de lettres inédites échangées par Alexandre Farnèse avec des membres de la famille Capizucchi (1572-1592)", in Bulletin de la Commission royale d'Histoire, XCV, (1931), pp. 73-92. 
Essen 1934: Léon van der Essen, Alexandre Farnèse, Prince de Parme, Gouverneur Général des Pays-Bas (1545-1592), II, (Bruxelles: Librairie Nationale d'art et d'histoire, 1934)

Essen 1936: Léon van der Essen, "Une critique de I'administration d'Alexandre Farnèse aux Pays-Bas par Don Juan de Idiáquez, Secrétaire d'Etat de Philippe II (1592)", in Bulletin de la Commission Royale d'Histoire, C, (1936), pp. 167-180.

Essen 1956: Léon van der Essen, "Croisade contre les hérétiques ou guerre contre des rebelles", in Revue d'histoire ecclésiastique, LI, (1956), pp. 4278.

Essen 2008: Léon van der Essen, El ejército español de Flandes: 1567-1584, (Yuste: Academia de Yuste, 2008)

Esteban Estríngana 2012: Alicia Esteban Estríngana, "Cabos de guerra: satisfacción de la ofialidad y eficacia bélica en el ejército de Flandes, entre los siglos XVI y XVII", in Los oficiales reales: los servidores del rey en la Monarquía Hispánica (siglos XVI-XVII), Juan Francisco Pardo Molero y Manuel Lomas Cortes (coords.), (Valencia: Universitat, 2012), pp. 265-293.

Fea 1886: Pietro Fea, Alessandro Farnese duca di Parma. Narrazione storica e militare, (Roma: Fratelli Bocca, 1886)

Filamondo 1694: Raffaele Maria Filamondo, Il genio bellicoso di Napoli. Memorie istoriche d'alcuni capitani napoletani, (Napoli: Parrino e Mutii, 1694)

Ghiglione 2005: Elda Ghiglione, Amedeo di Savoia Marchese di San Ramberto, (Firenze: Athenaeum, 2005)

González de León 2009: Fernando González de León, The Road to Rocroi. Class, Culture and Command in the Spanish Army of Flanders, 1567-1659, (Leiden-Boston: Brill, 2009)

Hanlon 1998: Gregory Hanlon, The Twilight of a military tradition. Italian Aristocracy and European Conflicts, 1560-1800, (New York: Holmes \& Meier, 1998)

Heuvel 1991: Charles van den Heuvel, "Papiere Bolwercken". De introductie van de italiaanse stede-en vestingbouw in de Nederlanden (1540-1609) en het gebruik van tekeningen, (Alphen aan den Rijn: Canaletto, 1991)

Hortal Muñoz 2011: José Eloy Hortal Muñoz, Los asuntos de Flandes. Las relaciones entre las Cortes de la Monarquía Hispana y de los Países Bajos durante el siglo XVI, (Saarbrücken: Editorial Académica Española, 2011) 
Lefèvre 1940-1960: Joseph Lefèvre, Correspondence de Philippe II sur les affaires des Pays-Bas, (Bruxelles: Palais des Academies, 1940-1960), 4 vols.

Maffi 2013: Davide Maffi, "Gli uomini del duca. Spunti biografici sull'ufficialità dell'Italia centrosettentrionale al servizio del Farnese", in Militari italiani dell'esercito di Alessandro Farnese nelle Fiandra, dir. Giuseppe Bertini, (Fidenza: Mattioli, 2013), pp. 107-135.

Muto 2007: Giovanni Muto, "Apparati militari e fabbisogno finanziario: il caso della Spagna "de los Austrias", in Militari e società civile nell'Europa dell'età moderna (secoli XVI-XVIII), dirs. Claudio Donati et Bernhard R. Kroener, (Bologna: Il Mulino, 2007), pp. 23-52.

Parker 1986: Geoffrey Parker, El ejército de Flandes y el Camino Español 1567-1659, (Madrid: Rialp, 1986)

Parker 2004: Geoffrey Parker, The army of Flanders and the Spanish Road 1567-1659, (Cambridge: Cambridge University Press, 2004)

Priuli 1861: "Relazione di Lorenzo Priuli", in dir. Eugenio Alberi, Le relazioni degli ambasciatori veneti al Senato, (Firenze: Società editrice fiorentina, 1861), pp. 242-250.

Riesco Terrero 1997: Ángel Riesco Terrero, "La ordenanza e instruccion del General Farnesio (Bruselas 1587)", in El ejército y la armada de Felipe II ante el IV centenario de su muerte (Madrid: Ministerio de Defensa, 1997), pp. 5786.

Rinaldi 1599: Liber relationum eorum quae gesta fuere in Belgio et alibi per serenissimum D. Ducem Alexandrum Farnesium, (Inedito, Bibliothèque Royale de Bruxelles, ms. II-1135, 1599)

Rivero Rodríguez 2004: Manuel Rivero Rodríguez, "La preeminencia del Consejo de Italia y el sentimento de la nación italiana", in La Monarquía de las naciones. Patria, nación y naturaleza en la Monarquía de España, dirs. Antonio Alvarez-Ossorio Alvariño, Bernardo José García García, (Madrid: Fundación Carlos de Amberes, 2004), pp. 483-503.

Rodríguez-Salgado 2016: María José Rodríguez-Salgado, "Kinship, collaboration and conflict: the complex relationship between Alessandro Farnese and Philip II", En la Corte del Rey de España. Liber Amicorum en homenaje a Carlos Gómez-Centurión Jiménez (1958-2011, dirs. Rafael Valladares, Feliciano Barrios e Juan Antonio Sánchez-Belén, (Madrid: Polifemo, 2016), pp. 61-105.

Sardi 1902: Cesare Sardi, I capitani lucchesi del sec. XVI, (Lucca: Tipografia Giusti, 1902) 
Sciarrini 2004: Marco Sciarrini, "La Italia natione". Il sentimento nazionale italiano in età moderna, (Milano: F. Angeli, 2004)

Signorotto 1998: Gianvittorio Signorotto, "Urbino dell'età di Filippo II", in Felipe II (1527-1598), Europa y la Monarquía Católica, ed. José Martínez Millan, (Madrid: Sociedad Estatal para la Conmemoración de los Centenarios de Felipe II y Carlos V, 1998), pp. 833-879.

Soen 2012: Violet Soen, "Reconquista and Reconciliation in the Dutch Revolt: The Campaign of Governor General Alessandro Farnese (1578-1592)", in Journal of Early Modern History, XVI, (2012), pp. 1-22.

Soranzo 1856: "Relazione di Francesco Soranzo", in Relazioni degli stati europei lette al Senato dagli ambasciatori veneti nel secolo decimosettimo, Spagna, dirs. Nicolò Barozzi e Guglielmo Berchet, (Venezia: P. Naratovich, 1856), I, pp. 27-214.

Spagnoletti 2004: Angelantonio Spagnoletti, "El concepto de naturaleza, nación y patria en Italia y el Reino de Nápoles con respecto a la monarquía de los Austrias", in La Monarquía de las naciones. Patria, nación y naturaleza en la Monarquía de España, dirs. Antonio Alvarez-Ossorio Alvariño, Bernardo José García García, (Madrid: Fundación Carlos de Amberes, 2004), pp. 505527.

Spagnoletti 2007: Angelantonio Spagnoletti, "Onore e spirito nazionale nei soldati italiani al servizio della monarchia spagnola", in Militari e società civile nell'Europa dell'età moderna (secoli XVI-XVIII), dirs. Claudio Donati et Bernhard R. Kroener, (Bologna: Il Mulino, 2007), pp. 211-253.

Spagnoletti 2009: Angelantonio Spagnoletti, "Le dinastie italiane e la guerra nelle Finadre", in Società e storia, 125, (2009), pp. 423-443.

Stirling-Maxwell 1883: Sir William Stirling-Maxwell, Don John of Austria, (London: Longmans-Green, 1883), 2 vols.

Strada 1638: Famiano Strada, Della guerra di Fiandra, Deca prima, (Roma: Facciotti, 1638)

Strada 1648: Famiano Strada, Della guerra di Fiandra, Deca seconda, (Roma: Eredi di Francesco, 1648)

Swart 2006: Erik Swart, "From "Landsknecht" to "Soldier": The Low German foot soldiers of the Low Countries in the second half of the Sixteenth Century", in International Review of Social History, LI, (2006), pp. 75-92. 
Tamalio 2013: Raffaele Tamalio, "I Gonzaga e le guerre di Fiandre", in Militari italiani dell'esercito di Alessandro Farnese nelle Fiandra, dir. Giuseppe Bertini, (Fidenza: Mattioli, 2013), pp. 137-151.

Tallon 2007: Alain Tallon (dir.), Le sentiment National dans I'Europe méridionale aux XVI et XVII siècles (France, Espagne, Italie), (Madrid: Casa de Velázquez, 2007)

Vázquez 1879: Alonso Vázquez, Los sucesos de Flandes y Francia del tiempo de Alejandro Farnese, (Madrid: Colección de documentos inéditos de la historia de España (CODOIN), 1879), 73.

Verdugo 1605: Francisco Verdugo, Li commentari delle cose successe in Frisia, (Napoli: Nella Stamperia di Felice Stigliola, 1605)

Visceglia 2001: Maria Antonietta Visceglia, "Non si ha da equiparare I'utile quando vi fosse I'honore". Scelte economiche e reputazione: intorno alle vendite dello stato feudale dei Caetani (1627)", in dir. Ead, La nobiltà romana in età moderna. Profili istituzionale e pratiche sociali, (Roma: Carocci, 2001), pp. 203-223. 